\title{
INTEGRABILITY CRITERIA FOR SYSTEMS OF NON- LINEAR PARTIAL DIFFERENTIAL EQUATIONS
}

\author{
HUBERT GOLDSCHMIDT
}

\section{Introduction}

In [5], we showed the existence of local solutions of analytic linear partial differential equations; in this paper, we generalize our previous result to an arbitrary analytic (nonlinear) system of partial differential equations and prove the Cartan-Kähler theorem for such a system formulated in terms of the jet bundles introduced by Ehresmann. The reader is strongly advised to read the proof of the linear Cartan-Kähler theorem (Theorem 4.1) of [5] as an introduction to the present paper, although this paper is essentially independent of [5].

We consider partial differential equations on fibered manifolds and do not define nonlinear equations in terms of differential operators as one can for linear equations (see [5]). In the nonlinear case this point of view would be too restrictive (see $\S 7$ ) and so we let arbitrary fibered submanifolds of jet bundles be partial differential equations, since many equations occurring in differential geometry are of this type (see E. Cartan [3]).

To generalize the methods of [5], where the jet bundles considered are vector bundles, we define affine bundles and analyse the structure of affine bundles which the jet bundles possess ( $\S \S 3$ and 5$)$. If every solution of order $k$ of a partial differential equation $R_{k}$ of order $k$ can be extended to a solution of order $k+1$, and if the solutions of order $k+1$ satisfy a regularity condition, the solutions of order $k+1$ form an equation $R_{k+1}$ of order $k+1$, the prolongation of $R_{k}$. This prolongation $R_{k+1}$ is actually an affine bundle over the equation $R_{k}$ whose affine bundle structure is induced by the affine bundle structure of a jet bundle. Under these conditions on $R_{k}$, this affine bundle structure of $R_{k+1}$ over $R_{k}$, together with the affine bundle structure of certain jet bundles (see Proposition 5.3), permits us to define the curvature $\kappa$ of the partial differential equation $R_{k}$. The curvature $\kappa$ is the obstruction to extending a solution of order $k+1$ to a solution of order $k+2$. Our definition of $k$ reduces for homogeneous linear equations to the definition of $\kappa$ given by Quillen [7], which is equivalent, with a change in sign, to the one Bott

Communicated by D. C. Spencer, June 2, 1967. This work was supported in part by the National Science Foundation. 
originally gave in [1], as was shown by Quillen [7]. In [5], we used still another definition of the curvature of a homogeneous linear equation.

We define the symbol of a partial differential equation and associate to it, following Spencer [8], certain cohomology groups $H^{k+l, j}$ whose vanishing, for $l \geq 0$, is equivalent to Cartan's notion of involutiveness, as was shown by Serre. A crucial property of the curvature $\kappa$ is the fact that it takes its values in a family of vector spaces induced by the Spencer cohomology group $H^{k, 2}$ (see Proposition 8.4). This permits us to determine, as in [5], the integrability conditions of the equation, which depend only on the formal solutions up to order $k+1$ and the second Spencer cohomology groups $H^{k+l, 2}$. Our formal existence theorems (Theorem 8.1 and Theorem 8.2), together with Spencer's estimate (see L. Ehrenpreis, V.W. Guillemin, and S. Sternberg [4] and W. J. Sweeney [10]) lead to the existence of analytic solutions for analytic equations and to generalizations of the Cartan-Kähler theorem and the Cartan-Kuranishi theorem (see M. Kuranishi [6]).

The author wishes to thank Professors V. W. Guillemin, M. Kuranishi, B. Morin, D. G. Quillen, and D. C. Spencer for many valuable suggestions concerning the various parts of this paper. Finally, the author would like to express his deep gratitude to Professor S. Sternberg for his constant encouragement and his invaluable advice during the preparation of this paper, which is essentially a part of the author's doctoral thesis presented to the Department of Mathematics, Harvard University, in partial fulfillment of the requirements for the degree of Doctor of Philosophy in the subject of Mathematics.

\section{Fibered manifolds}

Let $X$ be a differentiable manifold. (By differentiable, we shall always mean differentiable of class $C^{\infty}$.) We do not require that the dimensions of the different components of a differentiable manifold be the same. We shall denote by $T=T(X)$ the tangent bundle of $X$ and by $T^{*}=T^{*}(X)$ the cotangent bundle of $X$. By $S^{k} T^{*}, \wedge^{l} T^{*}, \otimes^{m} T^{*}$, we shall mean the $k$-th symmetric product of $T^{*}$, the $l$-th exterior product of $T^{*}$ and the tensor product of $m$ copies of $T^{*}$ respectively.

Definition 2.1. A fibered manifold $\pi: E \rightarrow X$ is a differentiable manifold $E$ together with a differentiable surjective map $\pi$ (projection) of $E$ onto $X$ of maximal rank such that the dimension of $E$ at $e \in E$ is greater than or equal to the dimension of $X$ at $\pi(e) \in X$.

We shall usually write simply $E$ to denote a fibered manifold $\pi: E \rightarrow X$. If $\pi: E \rightarrow X$ is a fibered manifold over $X$, we shall denote by $E_{x}=\pi^{-1}(x)$ the fiber of $E$ at $x \in X$, by $\mathscr{E}$ the sheaf of germs of differentiable sections of $E$ and by $\mathscr{E}_{x}$ the stalk of $\mathscr{E}$ at $x \in X$. If $s$ is a section of $E$ over an open set $U \subset X$ and $x \in X$, then $s_{x}$ is the germ of $s$ at $x$. If $Y$ is a submanifold of $X$, we denote by $E \mid Y$ the restriction of $E$ to $Y$, that is, the fibered manifold 
$\pi^{-1}(Y)$ over $Y$. Let $T(E)$ denote the tangent bundle of $E$ and $F(E)$ the subbundle of $T(E)$ consisting of all tangent vectors tangent to the fibers, that is, we have the exact sequence of vector bundles over $E$

$$
0 \longrightarrow F(E) \longrightarrow T(E) \stackrel{\pi_{*}}{\longrightarrow} \pi^{-1} T(X) \longrightarrow 0
$$

where $\pi^{-1} T(X)$ is the vector bundle over $E$ induced by $\pi$ from $T(X)$. By $O_{X}$ we denote $X$ considered as a fibered manifold over $X$ with projection the identity map of $X$.

Example. If $Y$ is any differentiable manifold, then $p r_{1}: X \times Y \rightarrow X$, where $p r_{1}$ is the projection onto the first factor, is a fibered manifold over $X$ called the trivial fibered manifold over $X$ with fiber $Y$. A section of this fibered manifold over an open set $U$ is simply the graph of a differentiable map from $U$ to $Y$.

Remark. Any fiber bundle and in particular any vector bundle is a fibered manifold over $X$.

If $\xi: W \rightarrow Y$ is a vector bundle over a differentiable manifold $Y$, then 0 is the zero section of $W$ over $Y$ and $O(y)$ is the zero vector of $W_{y}$. If $Z$ is another differentiable manifold, $f_{1}: Z \rightarrow X, f_{2}: Z \rightarrow Y$ are differentiable maps and if $\xi_{1}: W_{1} \rightarrow X, \xi_{2}: W_{2} \rightarrow Y$ are vector bundles, we shall denote by $W_{1} \otimes_{Z} W_{2}$ the vector bundle $f_{1}^{-1} W_{1} \otimes f_{2}^{-1} W_{2}$.

Throughout this paper, $\pi: E \rightarrow X, \pi^{\prime}: E^{\prime} \rightarrow X, \pi^{\prime \prime}: E^{\prime \prime} \rightarrow X$ denote fibered manifolds over $X$.

Definition 2.2. A morphism of fibered manifolds over $X$ from $\pi: E \rightarrow X$ to $\pi^{\prime}: E^{\prime} \rightarrow X$ is a differentiable map $\varphi: E \rightarrow E^{\prime}$ such that $\pi^{\prime} \circ \varphi=\pi$. We denote by $\varphi_{x}$ the map from $E_{x}$ to $E_{x}^{\prime}$, by $\varphi$ the sheaf map from $\mathscr{E}$ to $\mathscr{E}^{\prime}$ and by $\varphi_{*}$ the map from $T(E)$ to $T\left(E^{\prime}\right)$ or from $F(E)$ to $F\left(E^{\prime}\right)$ induced by $\varphi$.

Definition 2.3. We say that the sequence of fibered manifolds over $X$

$$
E \stackrel{\varphi}{\longrightarrow} E^{\prime} \stackrel{\varphi^{\prime}}{\longrightarrow} E^{\prime \prime}
$$

is exact if there exists a section $s^{\prime \prime}$ of $E^{\prime \prime}$ over $X$ such that:

(i) the sequence of sets

$$
E \stackrel{\varphi}{\longrightarrow} E^{\prime} \underset{s^{\prime \prime} \circ \pi^{\prime}}{\stackrel{\varphi^{\prime}}{\longrightarrow}} E^{\prime \prime}
$$

is exact;

(ii) the sequence of vector spaces

$$
F_{e}(E) \stackrel{\varphi_{*}}{\longrightarrow} F_{\varphi(e)}\left(E^{\prime}\right) \stackrel{\varphi_{* *}^{\prime}}{\longrightarrow} F_{\varphi^{\prime}(\varphi(e))}\left(E^{\prime \prime}\right)
$$

is exact, for every $e \in E$. 
We say that a morphism $\varphi: E \rightarrow E^{\prime}$ is a monomorphism if it is anbedding of manifolds, and is an epimorphism if $\varphi: E \rightarrow E^{\prime}$ is a fibered manifold. If $\varphi$ is both a monomorphism and an epimorphism, it is called an isomorphism. We say that the sequence

$$
O_{X} \longrightarrow E \stackrel{\varphi}{\longrightarrow} E^{\prime}
$$

is exact if $\varphi$ is a monomorphism and that the sequence

$$
E \stackrel{\varphi}{\longrightarrow} E^{\prime} \longrightarrow O_{X}
$$

is exact if $\varphi$ is an epimorphism. Note that these definitions are compatible with Definition 2.3.

The fibered product $E \times{ }_{X} E^{\prime}$ of $E$ and $E^{\prime}$ over $X$ consisting of all pairs $\left(e, e^{\prime}\right) \in E \times E^{\prime}$ satisfying $\pi(e)=\pi^{\prime}\left(e^{\prime}\right)$ is a differentiable manifold in a natural way and a fibered manifold over $X$. The projections $p r_{1}: E \times{ }_{X} E^{\prime} \rightarrow E$, $p r_{2}: E \times{ }_{X} E^{\prime} \rightarrow E^{\prime}$ sending $\left(e, e^{\prime}\right) \in E \times{ }_{X} E^{\prime}$ into $e, e^{\prime}$ respectively are epimorphisms of fibered manifolds over $X$.

Definition 2.4. A fibered manifold $\pi^{\prime}: E^{\prime} \rightarrow X$ is said to be a fibered submanifold of a fibered manifold $\pi: E \rightarrow X$ if $E^{\prime}$ is a subset of $E$ and if the inclusion of $E^{\prime}$ into $E$ is a monomorphism of fibered manifolds over $X$.

Definition 2.5. If $\varphi: E \rightarrow E^{\prime}$ is a morphism of fibered manifolds and $s^{\prime}$ is a section of $E^{\prime}$ over $X$, then $\operatorname{Ker}_{s^{\prime}} \varphi$, the kernel of $\varphi$ with respect to $s^{\prime}$, is the subset of $E$ consisting of all $e \in E$ satisfying $\varphi(e)=s^{\prime}(\pi(e))$.

Proposition 2.1. If $\varphi: E \rightarrow E^{\prime}$ is a morphism of fibered manifolds of locally constant rank, then the image of $\varphi$, denoted by $\operatorname{Im} \varphi$, is a fibered submanifold of $E^{\prime}$. If $s^{\prime}$ is a section of $E^{\prime}$ over $X$ satisfying $s^{\prime}(X) \subset \varphi(E)$, then $\operatorname{Ker}_{s^{\prime}} \varphi$ is a fibered submanifold of $E$ and the sequence

$$
O_{X} \longrightarrow \operatorname{Ker}_{s^{\prime}} \varphi \stackrel{i}{\longrightarrow} E \stackrel{\varphi}{\longrightarrow} \operatorname{Im} \varphi \longrightarrow O_{X}
$$

is exact, where $i$ is the inclusion of $\operatorname{Ker}_{s^{\prime}} \varphi$ into $E$.

The above proposition and the following lemma follow from the implicit function theorem in a straightforward way and their proofs will be omitted.

Lemma 2.1. If $\pi^{\prime}: E^{\prime} \rightarrow X$ is a fibered submanifold of $\pi: E \rightarrow X$, then, for each point $e \in E^{\prime}$, there exist an open neighborhood $V$ of $e$ in $E$, a fibered manifold $\zeta: Z \rightarrow U$ over $U=\pi V$, a section $z$ of $Z$ over $U$ and a morphism $\varphi: V \rightarrow Z$ of fibered manifolds over $U$ of constant rank such that $E^{\prime} \cap V$ $=\operatorname{Ker}_{z} \varphi$.

The statement of the following proposition is due to B. Mazur:

Proposition 2.2. Let $\pi^{\prime}: E^{\prime} \rightarrow X$ be a fibered submanifold of $\pi: E \rightarrow X$. Then there exist an open fibered submanifold $\pi^{\prime \prime}: E^{\prime \prime} \rightarrow X$ of $\pi: E \rightarrow X$ containing $E^{\prime}$, a fibered manifold $\zeta: Z \rightarrow X$, a section $Z$ of $Z$ over $X$ and $a$ 
morphism $\varphi: E^{\prime \prime} \rightarrow Z$ of fibered manifolds over $X$ of locally constant rank such that $E^{\prime}=\operatorname{Ker}_{2} \varphi$ if and only if the normal bundle of the imbedding of $E^{\prime}$ into $E$ is isomorphic to a vector bundle induced by $\pi^{\prime}$ from a vector bundle over $X$.

Proof. If the morphism $\varphi$ satisfying the above conditions exists, we may assume that $Z=\operatorname{Im} \varphi$ by Proposition 2.1. The normal bundle $N$ of the imbedding of $E^{\prime}$ into $E$ is isomorphic to $\varphi^{-1} N_{z}$, if $N_{z}$ denotes the normal bundle of the imbedding of $z(X)$ into $Z$. Since $E^{\prime}=\operatorname{Ker}_{z} \varphi$, the vector bundle $N$ is isomorphic to $\pi^{\prime-1}\left(z^{-1} N_{z}\right)$. Because $z$ is a section of $Z$ over $X$, the bundle $N$ satisfies the desired condition. Conversely, assume that there exists a vector bundle $\zeta: Z \rightarrow X$ such that $N$ is isomorphic to $\pi^{\prime-1} Z$. Because $N$ is isomorphic to the quotient of $F(E) \mid E^{\prime}$ by its sub-bundle $F\left(E^{\prime}\right)$, by the proof of the tubular neighborhood theorem, there exist an open fibered submanifold $\pi^{\prime \prime}: E^{\prime \prime} \rightarrow X$ of $E$ containing $E^{\prime}$ and an isomorphism $\psi: E^{\prime \prime} \rightarrow N$ of fibered manifolds over $X$ such that the diagram

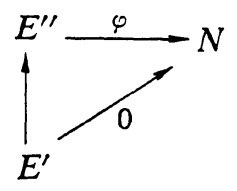

commutes, where 0 is the zero section of $N$. The natural epimorphism $p r_{2}$ of fibered manifolds over $X$ from $\pi^{\prime-1} Z=E^{\prime} \times{ }_{X} Z$ to $Z$ induces an epimorphism $\theta$ of fibered manifolds over $X$ from $N$ to $Z$. Define $\varphi: E^{\prime \prime} \rightarrow Z$ to be the composition $\theta \circ \phi$ and $z$ to be the zero section of $Z$; clearly $\varphi$ has the required properties.

\section{Affine bundles}

We begin by recalling certain properties of affine spaces. Let $k$ be a field. By an affine space $A$ over $k$ modeled on a vector space $T$ over $k$, we mean a homogeneous space of the additive group of $T$ on which $T$ acts freely. We denote by $t+a$ the image of $a \in A$ under the action of $t \in T$, and if $a, b \in A$, by $a-b$ the unique element of $T$ such that $(a-b)+b=a$. For each $a \in A$, we have a natural identification of $A$ with $T$ sending $x \in A$ into $x-a \in T$ which induces on $A$ the structure of a vector space over $k$ with origin $a$. A non-empty subset $V$ of $A$ is an affine subspace of $A$ if $V$ is a subspace of $A$ considered as a vector space with origin a point of $V$; the set $D$ of all elements $x-y$ of $T$, with $x, y$ belonging to $V$, is a linear subspace of $T$ and $V$ is an affine space modeled on $D$ in a canonical way. If $A^{\prime}$ is an affine space modeled on a vector space $T^{\prime}$ over $k$, by a morphism $\varphi: A \rightarrow A^{\prime}$ of affine spaces over $k$, we mean a map preserving barycenters. For any such morphism, there exists a unique linear map $\psi: T \rightarrow T^{\prime}$ such that 


$$
\varphi(t+x)=\varphi(t)+\varphi(x)
$$

for all $x \in A, t \in T$, which we call the linear map associated to $\varphi$ (see N. Bourbaki [2]).

If $\varphi: A \rightarrow A^{\prime}$ is a morphism of affine spaces whose associated linear map is $\phi: T \rightarrow T^{\prime}$, we define an equivalence relation on $A^{\prime}$ : we say that $a^{\prime} \in A^{\prime}$ is equivalent to $b^{\prime} \in A^{\prime}$ if and only if $a^{\prime}-b^{\prime} \in \psi(T)$. We denote the set of equivalence classes by Coker $\varphi$ and by $\rho$ the natural projection of $A^{\prime}$ onto Coker $\varphi$. Note that $\rho \varphi(a)=\rho \varphi(b)$, if $a, b \in A$, so that $\rho \varphi(A)$ is a distinguished point of Coker $\varphi$. It is easily verified that Coker $\varphi$ is an affine space modeled on the cokernel of $\phi$, Coker $\psi$, and that $\rho$ is a morphism of affine spaces whose associated linear map is the natural projection of $T^{\prime}$ onto Coker $\psi$. Since Coker $\varphi$ has a distinguished element $\rho \varphi \cdot(A)$, it can be identified in a natural way with Coker $\psi$. We call Coker $\varphi$ the cokernel of $\varphi$.

If $i: V \rightarrow A$ is the inclusion of an affine subspace $V$ of $A$ into $A$, the cokernel of $i$ will be called the quotient $A / V$ of $A$ by $V$.

If $a^{\prime} \in A^{\prime}$, we denote by $\operatorname{Ker}_{a}, \varphi$ the inverse image under $\varphi$ of $a^{\prime}$. If $\operatorname{Ker}_{a^{\prime}} \varphi$ is non-empty, it is easily seen that it is an affine subspace of $A$ modeled on the kernel of $\phi, \operatorname{Ker} \psi$.

Definition 3.1. An affine bundle $A$ over $X$ modeled on a vector bundle $\xi: W \rightarrow X$ is a fibered manifold $\pi: A \rightarrow X$ together with a morphism of fibered manifolds over $X$

$$
W \times{ }_{X} A \rightarrow A
$$

sending ( $w, a) \in W \times{ }_{X} A$ into $w+a \in A$, such that, for each $x \in X$, the fiber $A_{x}$ is an affine space modeled on the vector space $W_{x}$ under the action of $W_{x}$ on $A_{x}$ sending $(w, a) \in W_{x} \times A_{x}$ into $w+a \in A_{x}$.

If $a \in A_{x}$, the map $w \rightarrow w+a$ of $W_{x}$ into $A_{x}$ is a diffeomorphism. If $b \in A_{x}$, the map of $A_{x}$ into $W_{x}$ sending $a$ into $a-b$ is also a diffeomorphism.

If $s$ is a section of $A$ over an open set $U \subset X$, the map of $A \mid U$ into $W \mid U$ sending $a$ into $a-s(\pi(a))$ is an isomorphism of fibered manifolds over $U$. Therefore $A$ is locally trivial, that is, given $x \in X$, there exist a neighborhood $V$ of $x$ and an isomorphism $\varphi: A \mid V \rightarrow V \times A_{x}$ of fibered manifolds over $V$.

Note that $O_{X}$ is an affine bundle over $X$ modeled on the zero-dimensional vector bundle over $X$.

Definition 3.2. If $\pi_{1}: E_{1} \rightarrow E, \pi_{1}^{\prime}: E_{1}^{\prime} \rightarrow E^{\prime}$ are fibered manifolds, then a differentiable map $\varphi_{1}: E_{1} \rightarrow E_{1}^{\prime}$ is said to be a morphism of fibered manifolds from $E_{1}$ to $E_{1}^{\prime}$ over a morphism $\varphi: E \rightarrow E^{\prime}$ of fibered manifolds over $X$ if $\pi_{1}^{\prime} \circ \varphi_{1}=\varphi \circ \pi_{1}$. If $E_{1}, E_{1}^{\prime}$ are vector (respectively affine) bundles over $E, E^{\prime}$ respectively and if the map from $\left(E_{1}\right)_{e}$ to $\left(E_{1}^{\prime}\right)_{\varphi(e)}$ induced by $\varphi_{1}$ is a linear map of vector spaces (respectively morphism of affine spaces) for each $e \in E$, then we say that $\varphi_{1}$ is a morphism of vector (respectively affine) bundles from $E_{1}$ to 
$E_{1}^{\prime}$ over the morphism $\varphi$. A morphism $\varphi_{1}: E_{1} \rightarrow E_{1}^{\prime}$ of affine bundles over a morphism $\varphi: E \rightarrow E^{\prime}$ of fibered manifolds over $X$ is said to be a monomorphism of affine bundles if $\varphi_{1}$ is a monomorphism of fibered manifolds over $X$. If $E=E^{\prime}$ and $\varphi$ is the identity map of $E$, then we say that a morphism of fibered manifolds from $E_{1}$ to $E_{1}^{\prime}$ over $\varphi$ is a morphism of fibered manifolds over $E$.

Proposition 3.1. If $\alpha: A \rightarrow E, \alpha^{\prime}: A^{\prime} \rightarrow E^{\prime}$ are affine bundles over $E, E^{\prime}$ modeled on vector bundles $\xi: W \rightarrow E, \xi^{\prime}: W^{\prime} \rightarrow E^{\prime}$ respectively and if $\varphi: A \rightarrow A^{\prime}$ is a morphism of affine bundles over a morphism $\tilde{\varphi}: E \rightarrow E^{\prime}$, there exists a unique morphism of vector bundles $\phi: W \rightarrow W^{\prime}$ over $\tilde{\varphi}$ such that

$$
\varphi(w+a)=\phi(w)+\varphi(a)
$$

for all $a \in A, w \in W$, with $\alpha(a)=\xi(w)$.

We say that $\phi$ is the morphism of vector bundles associated to $\varphi$. Given a morphism $\psi: W \rightarrow W^{\prime}$ of vector bundles over a morphism $\tilde{\varphi}: E \rightarrow E^{\prime}$ and sections $s, s^{\prime}$ of $A, A^{\prime}$ over $E, E^{\prime}$ respectively, the map

$$
a \longmapsto \phi(a-s(\alpha(a)))+s^{\prime}(\tilde{\varphi}(\alpha(a)))
$$

is a morphism of affine bundles from $A$ to $A^{\prime}$ over $\tilde{\varphi}$.

The following proposition is easily verified:

Proposition 3.2. Let $A, A^{\prime}, A^{\prime \prime}$ be affine bundles over $X$ modeled on vector bundles $W, W^{\prime}, W^{\prime \prime}$ over $X$. Then the sequence of morphisms of affine bundles over $X$

$$
A \stackrel{\varphi}{\longrightarrow} A^{\prime} \stackrel{\varphi^{\prime}}{\longrightarrow} A^{\prime \prime}
$$

is exact if and only if the associated sequence of morphisms of vector bundles over $X$

$$
W \stackrel{\varphi}{\longrightarrow} W^{\prime} \stackrel{\phi^{\prime}}{\longrightarrow} W^{\prime \prime}
$$

is exact.

Definition 3.3. A locally trivial fibered submanifold $\pi^{\prime}: A^{\prime} \rightarrow X$ of an affine bundle $\pi: A \rightarrow X$ is said to be an affine sub-bundle of $A$ if $A_{x}^{\prime}$ is an affine subspace of $A_{x}$, for each $x \in X$.

An affine sub-bundle $\pi^{\prime}: A^{\prime} \rightarrow X$ of an affine bundle $\pi: A \rightarrow X$ modeled on a vector bundle $\xi: W \rightarrow X$ is an affine bundle modeled on a sub-bundle $W^{\prime}$ of $W$. Indeed, for each $x \in X$, the subset $A_{x}^{\prime}$ of $A_{x}$ is an affine space modeled on the subspace $W_{x}^{\prime}$ of $W_{x}$ consisting of all elements $a^{\prime}-b^{\prime}$, with $a^{\prime}, b^{\prime} \in A_{x}^{\prime}$. Since $A^{\prime}$ is locally trivial,

$$
W^{\prime}=\bigcup_{x \in X} W_{x}^{\prime}
$$

is a sub-bundle of $W$ on which $A^{\prime}$ is modeled.

If $\pi^{\prime}: A^{\prime} \rightarrow X$ is a fibered submanifold of $\pi: A \rightarrow X$, if $A_{x}^{\prime}$ is an affine subspace of $A_{x}$ for each $x \in X$, and if 


$$
W^{\prime}=\bigcup_{x \in X} W_{x}^{\prime}
$$

is a sub-bundle of $W$, then $A^{\prime}$ is an affine sub-bundle of $A$.

Proposition 3.3. Let $\pi: A \rightarrow X, \pi^{\prime}: A^{\prime} \rightarrow X$ be affine bundles over $X$ modeled on vector bundles $\xi: W \rightarrow X, \xi^{\prime}: W^{\prime} \rightarrow X$. Then a morphism $\varphi: A \rightarrow A^{\prime}$ of affine bundles over $X$ is of locally constant rank if and only if the linear map $\psi_{x}: W_{x} \rightarrow W_{x}^{\prime}$, with $x \in X$, induced by the morphism of vector bundles associated to $\varphi$, has locally constant rank.

The proof of this proposition will be left to the reader.

If $\varphi: A \rightarrow A^{\prime}$ is a morphism of affine bundles over $X$, then for $x \in X$, define $(\operatorname{Coker} \varphi)_{x}=\operatorname{Coker} \varphi_{x}$, and let

$$
\text { Coker } \varphi=\bigcup_{x \in X}(\operatorname{Coker} \varphi)_{x} .
$$

If $A, A^{\prime}$ are modeled on vector bundles $W, W^{\prime}$ over $X$, we can canonically identify the affine space Coker $\varphi_{x}$ with the vector space on which it is modeled, Coker $\psi_{x}$, where $\phi$ is the morphism of vector bundles associated to $\varphi$, and hence also Coker $\varphi$ with Coker $\phi$. If $\varphi$ has locally constant rank, then so does $\psi$ by Proposition 3.3, so that Coker $\psi$ is a vector bundle over $X$; we can give Coker $\varphi$ the structure of fibered manifold over $X$ induced from the structure of fibered manifold of Coker $\psi$ via the above identification. Then Coker $\varphi$ becomes an affine bundle modeled on Coker $\psi$. If $\rho_{x}$ is the natural projection of $A_{x}^{\prime}$ onto $(\operatorname{Coker} \varphi)_{x}$, we obtain a map $\rho: A^{\prime} \rightarrow \operatorname{Coker} \varphi$ which is easily seen to be a morphism of affine bundles whose associated morphism of vector bundles is the natural projection $\sigma: W^{\prime} \rightarrow$ Coker $\phi$. Furthermore,

$$
A \stackrel{\varphi}{\longrightarrow} A^{\prime} \stackrel{\rho}{\longrightarrow} \text { Coker } \varphi \longrightarrow O_{X}
$$

is an exact sequence of affine bundles over $X$ by Proposition 3.2, since

$$
W \stackrel{\phi}{\longrightarrow} W^{\prime} \stackrel{\sigma}{\longrightarrow} \text { Coker } \psi \longrightarrow 0
$$

is an exact sequence of vector bundles over $X$. Therefore, we have proved

Proposition 3.4. If $\varphi: A \rightarrow A^{\prime}$ is a morphism of affine bundles over $X$ of locally constant rank, then there exist an affine bundle Coker $\varphi$, the cokernel of $\varphi$, and a morphism of affine bundles $\rho: A \rightarrow$ Coker $\varphi$ such that the sequence

$$
A \stackrel{\varphi}{\longrightarrow} A^{\prime} \stackrel{\rho}{\longrightarrow} \operatorname{Coker} \varphi \longrightarrow O_{X}
$$

is exact. Moreover, Coker $\varphi$ can be identified in a natural way with the cokernel of the morphism of vector bundles associated to $\varphi$.

The above proposition can be applied to the inclusion $i$ of an affine sub- 
bundle $A^{\prime}$ of $A$ into $A$, which is a monomorphism of affine bundles; the cokernel of $i$ will be called the quotient $A / A^{\prime}$ of $A$ by $A^{\prime}$.

Proposition 3.5. Let $\alpha: A \rightarrow E, \alpha^{\prime}: A^{\prime} \rightarrow E^{\prime}$ be affine bundles over $E, E^{\prime}$ modeled on vector bundles $\xi: W \rightarrow E, \xi^{\prime}: W^{\prime} \rightarrow E^{\prime}$ and let $\varphi$ be a morphism of affine bundles, over a morphism $\tilde{\varphi}: E \rightarrow E^{\prime}$ of locally constant rank, whose associated morphism of vector bundles is a map $\psi: W \rightarrow W^{\prime}$. Let $s^{\prime}$ be a section of $A^{\prime}$ over $X$ satisfying $s^{\prime}(X) \subset \varphi(A)$; setting $K=\operatorname{Ker}_{s^{\prime}} \varphi, \bar{K}=$ $\operatorname{Ker}_{\alpha^{\prime} \circ s^{\prime}} \tilde{\varphi}$, assume that $\alpha: K \rightarrow \bar{K}$ is surjective. If the restriction of $\phi$ to $W \mid \bar{K}$ has locally constant rank, then $K$ is an affine sub-bundle of $A \mid \bar{K}$ over $\bar{K}$ modeled on $(\operatorname{Ker} \psi) \mid \bar{K}$ and the restriction of $\varphi$ to $A \mid \bar{K}$ has locally constant rank.

Proof. Since $s^{\prime}(X) \subset \varphi(A)$, we have $\left(\alpha^{\prime} \circ s^{\prime}\right)(X) \subset \tilde{\varphi}(E)$ and Proposition 2.1 applies; hence $\bar{K}$ is a fibered submanifold of $\pi: E \rightarrow X$. Since $\varphi$ is a morphism of affine bundles over $\tilde{\varphi}$, for each $e \in \bar{K}$, the map $\varphi_{e}: A_{\epsilon} \rightarrow A_{\tilde{\varphi}(e)}^{\prime}$ is a morphism of affine spaces. Because $s^{\prime}(\pi(e))$ belongs to $A_{\tilde{\varphi}(e)}^{\prime}$ and because $\alpha: K \rightarrow \bar{K}$ is surjective, $\operatorname{Ker}_{s^{\prime}(\pi(e))} \varphi_{e}$ is an affine subspace of $A_{e}$ modeled on $\operatorname{Ker} \psi_{e}$. Since $(\operatorname{Ker} \psi) \mid \bar{K}$ is a sub-bundle of $W \mid \bar{K}$, to show that $K$ is an affine sub-bundle of $A \mid \bar{K}$, it is sufficient to show that for every $e \in \bar{K}$, there exists a section of $A \mid \bar{K}$ over a neighborhood of $e \in \bar{K}$ whose image lies in $K$. We first note that the vector bundle $\operatorname{Im}(\psi \mid(W \mid \bar{K}))$ is the subset of $W^{\prime}$ consisting of all elements of $W^{\prime}$ of the form $\varphi(a)-s^{\prime}(\pi(a))$, where $a \in A \mid \bar{K}$. Indeed, given $w \in W \mid \bar{K}$, there exists $a \in K$, with $\alpha(a)=\xi(w)$; we have $\varphi(w+a)$ $=\phi(w)+s^{\prime}(\pi(a))$ and so $\phi(w)=\varphi(w+a)-s^{\prime}(\pi(a))$. Given $a \in A \mid \bar{K}$, choose $a^{\prime} \in K$ with $\alpha\left(a^{\prime}\right)=\alpha(a)$; then

$$
\varphi(a)-s^{\prime}(\pi(a))=\varphi(a)-\varphi\left(a^{\prime}\right)=\psi\left(a-a^{\prime}\right) .
$$

Now choose a morphism of vector bundles $\sigma: \tilde{\varphi}^{-1}\{\operatorname{Im}(\phi \mid(W \mid \bar{K}))\} \rightarrow W \mid \bar{K}$ over $\bar{K}$ such that $\phi \circ \sigma=$ id. Given an element $e \in \bar{K}$, choose a section $s$ of $A \mid \bar{K}$ over a neighborhood $V$ of $e \in \bar{K}$. Clearly $\varphi \circ s$ and $s^{\prime} \circ \pi$ are mappings of $V$ into $A^{\prime}$ satisfying $\alpha^{\prime} \circ(\varphi \circ s)=\alpha^{\prime} \circ\left(s^{\prime} \circ \pi\right)$. Therefore these maps induce a map $s^{\prime} \circ \pi-\varphi \circ s$ from $V$ to $W^{\prime}$ such that $\xi^{\prime}\left(s^{\prime} \circ \pi-\varphi \circ s\right)=\tilde{\varphi}$. By the preceding remarks, the section

$$
v \mapsto \sigma\left(v,\left(s^{\prime} \circ \pi-\varphi \circ s\right)(v)\right)
$$

of $W$ over $V$ is well-defined and will denoted by $\sigma\left(s^{\prime} \circ \pi-\varphi \circ s\right)$; the section

$$
\bar{s}=\sigma\left(s^{\prime} \circ \pi-\varphi \circ s\right)+s
$$

of $A$ over $V$ is also well-defined. We have

$$
\varphi \circ \bar{s}=\phi\left(\sigma\left(s^{\prime} \circ \pi-\varphi \circ s\right)\right)+\varphi \circ s=\left(s^{\prime} \circ \pi-\varphi \circ s\right)+\varphi \circ s=s^{\prime} \circ \pi .
$$

Hence the image of the section $\bar{s}$ lies in $K$ and $\alpha: K \rightarrow \bar{K}$ is an affine bundle. Moreover, for $a \in A \mid V$ 


$$
\varphi(a)=\phi(a-\bar{s}(\alpha(a)))+s^{\prime}(\pi(\alpha(a)))
$$

which implies that $\varphi \mid(A \mid \bar{K})$ has locally constant rank.

Proposition 3.6. If $\pi: A \rightarrow X$ is an affine bundle over $X$ modeled on a vector bundle $\xi: W \rightarrow X$, and if $\pi^{-1} W$ denotes the vector bundle over $A$ induced from $W$ by $\pi$, there is a natural isomorphism $\mu$ of vector bundles over $A$ from $\pi^{-1} W$ to $F(A)$.

Proof. An element $w \in W_{x}$, with $x \in X$, determines a one-parameter family of diffeomorphisms $\mu_{t}(w)$ of $A_{x}$, by setting $\mu_{t}(w)(b)=t w+b$, for all $b \in A_{x}$, $t \in R$. Then

$$
\tilde{w}=\left.\frac{d}{d t} \mu_{t}(w)\right|_{t=0}
$$

is a vector field on $A_{x}$, and since $A_{x}$ is a submanifold of $A$, for each $b \in A_{x}$, $\tilde{w}(b)$ is an element of $F_{b}(A)$. Consider the map $\mu$ sending $(a, w) \in \pi^{-1} W$, with $a \in A_{x}, w \in W_{x}$, for some $x \in X$, into $\tilde{w}(a) \in F(A)$. Then $\mu$ is clearly a monomorphism of vector bundles over $A$, since if $\tilde{w}(a)=0$, then $\mu_{t}(w)(a)=a$ for some $t \neq 0$ and $w=0(x)$. Because the dimensions of these two vector bundles are the same, $\mu$ is actually an isomorphism.

Proposition 3.7 (Naturality of $\mu$ ). If $\alpha: A \rightarrow E, \alpha^{\prime}: A^{\prime} \rightarrow E^{\prime}$ are affine bundles over $E, E^{\prime}$ modeled on vector bundles $\xi: W \rightarrow E, \xi^{\prime}: W^{\prime} \rightarrow E^{\prime}$ and if $\varphi: A \rightarrow A^{\prime}$ is a morphism of affine bundles over a morphism $\tilde{\varphi}: E \rightarrow E^{\prime}$, whose associated morphism of vector bundles is a map $\psi: W \rightarrow W^{\prime}$, then the diagram of vector bundles over $A$

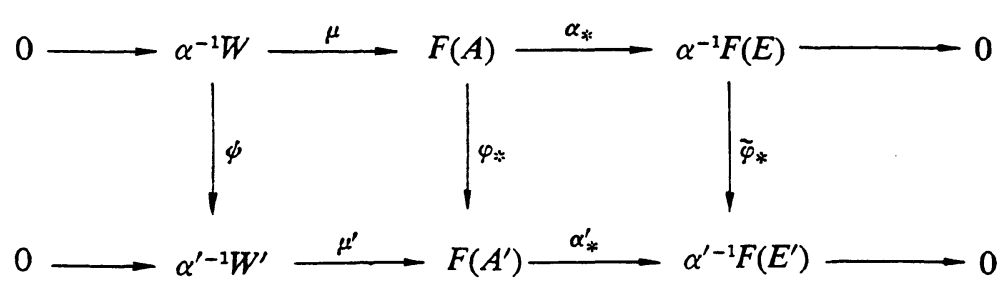

is commutative and has exact rows, where $\phi: \alpha^{-1} W \rightarrow \alpha^{-1} W^{\prime}, \tilde{\varphi}_{*}: \alpha^{-1} F(E) \rightarrow$ $\alpha^{\prime-1} F\left(E^{\prime}\right)$ are morphisms of vector bundles over $\tilde{\varphi}$ induced by $\phi: W \rightarrow W^{\prime}$, $\tilde{\varphi}_{*}: F(E) \rightarrow F\left(E^{\prime}\right)$ respectively.

Proof. The exactness of the rows of (3.1) follows from Proposition 3.6. If $e \in E, w \in W_{e}$, we have

$$
\varphi \circ\left(\mu_{t}(w)(a)\right)=\varphi(t w+a)=t \psi(w)+\varphi(a)=\mu_{t}^{\prime}(\phi(w))(\varphi(a))
$$

for all $a \in A_{e}$. The proposition is an easy consequence of this identity. 


\section{Jet bundles as fibered manifolds}

Let $s$ be a section of the fibered manifold $E$ over a neighborhood of $x \in X$. The equivalence class of all sections of $E$ agreeing with $s$ to order $k$ at $x$ is called the $k$-jet of $s$ at $x$ and is denoted by $j_{k}(s)(x)$. The point $x \in X$ is called the source of the jet $j_{k}(s)(x)$, and $s(x)$ its target. Let $J_{k}(E)_{x}$ denote the set of all $k$-jets at $x$ of sections of $E$ and let

$$
J_{k}(E)=\bigcup_{x \in X} J_{k}(E)_{x}
$$

We call $J_{k}(E)$ the bundle of $k$-jets of $E$. For $k \geq l$, we denote by $\pi_{l}: J_{k}(E) \rightarrow$ $J_{l}(E)$ the map sending a $k$-jet into the $l$-jet it determines. In particular, the map $\pi_{0}: J_{k}(E) \rightarrow E$ sends a $k$-jet into its target, so we shall identify $J_{0}(E)$ with $E$.

Proposition 4.1. There exists a natural differentiable manifold structure on $J_{k}(E)$. In fact, $J_{k}(E)$ is a fibered manifold over $X$ whose projection $\pi: J_{k}(E) \rightarrow X$ sends a $k$-jet into its source; moreover $\pi_{l}: J_{k}(E) \rightarrow J_{l}(E)$ is an epimorqhism of fibered manifolds over $X$, for $k \geq l$.

If $s$ is a section of $E$ over an open set $U \subset X$, the map $y \rightarrow j_{k}(s)(y)$ of $U$ into $J_{k}(E)$ is a section $j_{k}(s)$ of $J_{k}(E)$ over $U$, and the map $s \mapsto j_{k}(s)$ induces a morphism of sheaves $j_{k}: \mathscr{E} \rightarrow \mathscr{J}_{k}(\mathscr{E})$.

Note that the fibered manifold $J_{k}\left(O_{X}\right)$ is isomorphic to $O_{X}$ and will be identified with $O_{X}$, and that the fibered manifold $J_{k}\left(E \times{ }_{X} E^{\prime}\right)$ is naturally isomorphic to $J_{k}(E) \times{ }_{X} J_{k}\left(E^{\prime}\right)$. If $E$ is a vector bundle, then $J_{k}(E)$ has the structure of a vector bundle over $X$. If $U$ is an open subset of $X$, then the restriction $J_{k}(E) \mid U$ of $J_{k}(E)$ to $U$ is isomorphic to the bundle $J_{k}(E \mid U)$ of $k$-jets of $E \mid U$. Furthermore, if $E$ is the trivial fibered manifold over $X$ with fiber a differentiable manifold $Y$, then $J_{k}(E)$ is diffeomorphic to the manifold of $k$-jets of (local) differentiable maps of $X$ into $Y$ in the sense of Ehresmann.

Let $\varphi: J_{k}(E) \rightarrow E^{\prime}$ be a morphism of fibered manifolds over $X$. A sheaf morphism of the form $\varphi \circ j_{k}: \mathscr{E} \rightarrow \mathscr{E}^{\prime}$ is called a differential operator from $E$ to $E^{\prime}$ of order $k$. The $l$-th prolongation $p_{l}(\varphi): J_{k+l}(E) \rightarrow J_{l}\left(E^{\prime}\right)$ of $\varphi$ is defined to be the unique morphism of fibered manifolds over $X$ such that the diagram

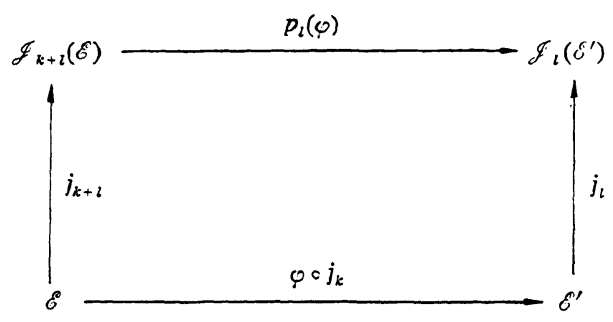

commutes. In particular, the $l$-th prolongation of the identity map $\operatorname{id}_{k}$ of $J_{k}(E)$ 
is a monomorphism of fibered manifolds $p_{l}\left(\mathrm{id}_{k}\right): J_{k+l}(E) \rightarrow J_{l}\left(J_{k}(E)\right)$. We shall identify $J_{k+l}(E)$ with its image in $J_{l}\left(J_{k}(E)\right)$ under the natural monomorphism $p_{l}\left(\mathrm{id}_{k}\right)$. The map $\varphi$ also induces a morphism of fibered manifolds $J_{l}(\varphi): J_{l}\left(J_{k}(E)\right) \rightarrow J_{l}\left(E^{\prime}\right)$ such that the diagram

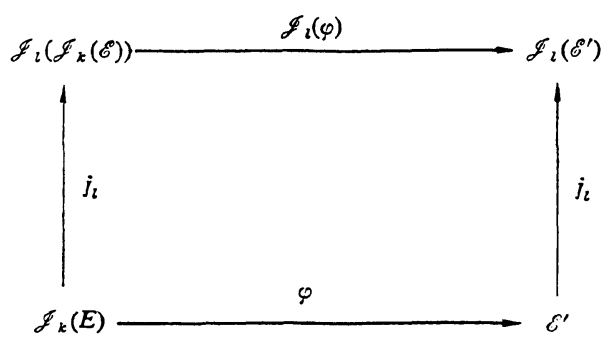

commutes, where $\mathscr{J}_{2}(\varphi)$ is the map of sheaves induced by $J_{l}(\varphi)$.

Proposition 4.2. If $\varphi: J_{k}(E) \rightarrow E^{\prime}, \varphi^{\prime}: J_{l}\left(E^{\prime}\right) \rightarrow E^{\prime \prime}$ are morphisms of fibered manifolds over $X$, then

$$
p_{m}\left(\varphi^{\prime} \circ p_{l}(\varphi)\right)=p_{m}\left(\varphi^{\prime}\right) \circ p_{l+m}(\varphi), \quad \text { for } m \geq 0 .
$$

The proposition follows directly from the definitions of the maps involved.

Proposition 4.3. If $\varphi: J_{k}(E) \rightarrow E^{\prime}$ is a morphism of fibered manifolds, then the diagram

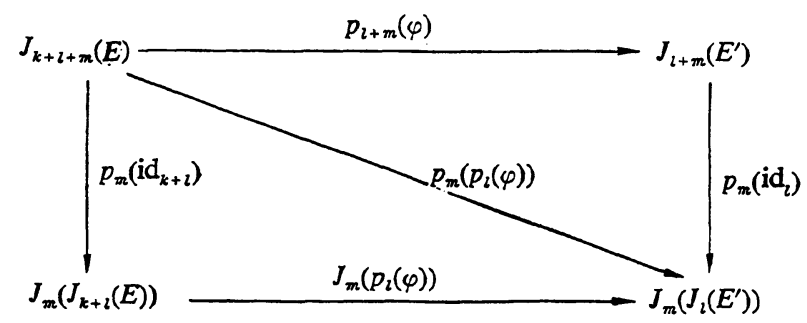

commutes.

Proof. It follows directly from the definitions of the maps involved that the following diagrams commute:

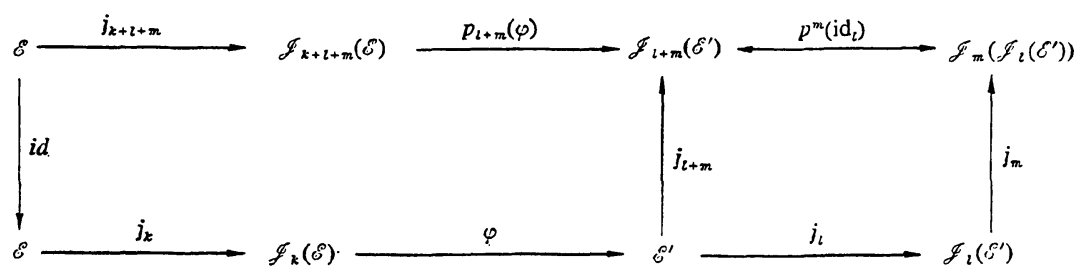



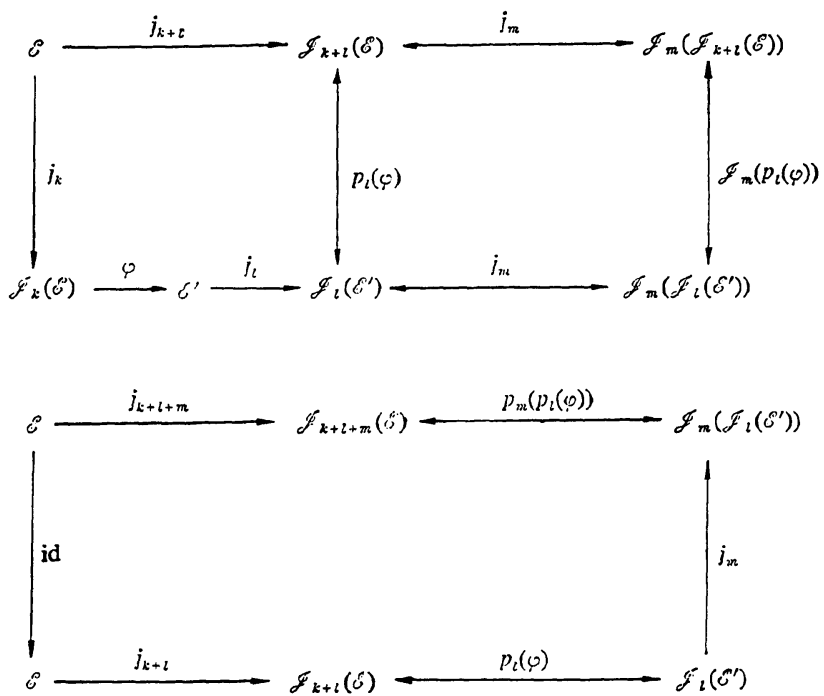

Therefore the diagram

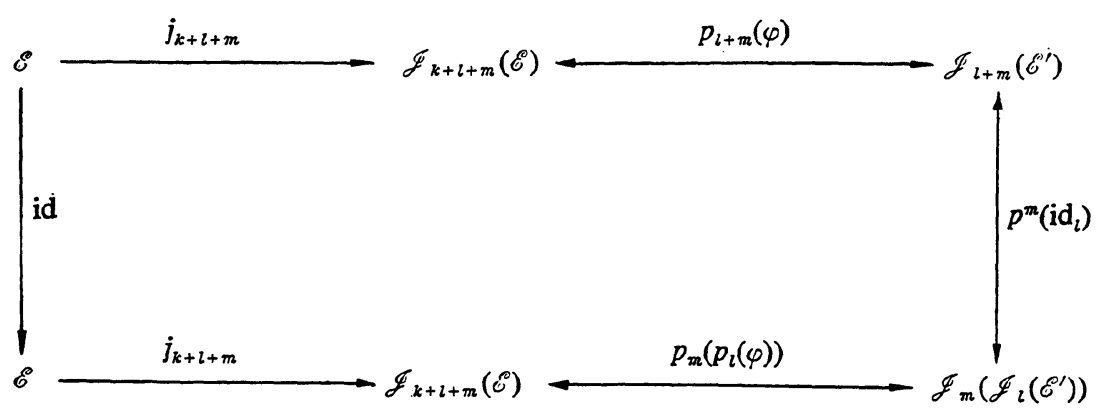

and the outer diagram of

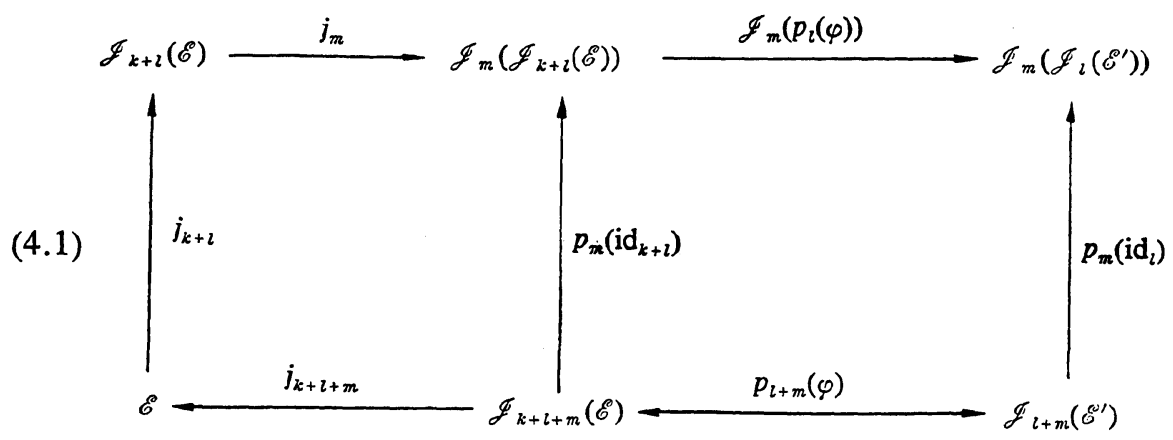


also commute. However the first square of (4.1) commutes by the definition of the map $p_{m}\left(\mathrm{id}_{k+l}\right)$; hence

$$
\begin{aligned}
p_{m}\left(p_{l}(\varphi)\right) \circ j_{k+l+m} & =p_{m}\left(\mathrm{id}_{l}\right) \circ p_{l+m}(\varphi) \circ j_{k+l+m} \\
& =\mathscr{J}_{m}\left(p_{l}(\varphi)\right) \circ p_{m}\left(\mathrm{id}_{k+l}\right) \circ j_{k+l+m}
\end{aligned}
$$

as maps from $\mathscr{E}$ to $\mathscr{J}_{m}\left(\mathscr{J}_{l}\left(\mathscr{E}^{\prime}\right)\right)$ and so the proposition is established.

If $F$ is an open fibered submanifold of $J_{k}(E)$ and $\varphi: F \rightarrow E^{\prime}$ is a morphism of fibered manifolds over $X$, then one can define the $l$-th prolongation $p_{l}(\varphi)$ as a morphism of fibered manifolds from the open fibered submanifold $\pi_{k}^{-1} F$ of $J_{k+\imath}(E)$ to $J_{l}\left(E^{\prime}\right)$ as follows. It is the unique morphism such that

$$
p_{l}(\varphi)\left(j_{k+l}(s)(x)\right)=j_{l}\left(\varphi \circ j_{k}(s)\right)(x)
$$

for all sections $s$ of $E$ over a neighborhood of $x \in X$ satisfying $j_{k}(s)(x) \in F$. Note that $p_{l}(\varphi)$ is well-defined, since, for any such section $s$ of $E$, it follows, from the fact that $F$ is an open subset of $J_{k}(E)$, that $j_{k}(s)(y)$ belongs to $F$ for all $y$ in a neighborhood of $x$. It is easily seen that the analogues of Propositions 4.2, 4.3 hold for such $\varphi$.

Proposition 4.4. Let $\pi_{1}: E_{1} \rightarrow E, \pi_{1}^{\prime}: E_{1}^{\prime} \rightarrow E$ be fibered manifolds over $E$ and let $\varphi: E_{1} \rightarrow E_{1}^{\prime}$ be a morphism of fibered manifolds over $E$ of locally constant rank. Then $p_{k}(\varphi): J_{k}\left(E_{1}\right) \rightarrow J_{k}\left(E_{1}^{\prime}\right)$ is a morphism over $J_{k}(E)$ of locally constant rank. If $\varphi$ is a monomorphism (respectively an epimorphism) over $E$, then $p_{k}(\varphi)$ is a monomorphism (respectively an epimorphism) over $J_{k}(E)$. If $s_{1}^{\prime}$ is a section of $E_{1}^{\prime}$ over $E$ satisfying $s_{1}^{\prime}(E) \subset \varphi\left(E_{1}\right)$, then $p_{k}\left(s_{1}^{\prime}\right)\left(J_{k}(E)\right) \subset$ $p_{k}(\varphi)\left(J_{k}\left(E_{1}\right)\right)$ and the sequence

$$
O_{J_{k}}(E) \longrightarrow J_{k}\left(\operatorname{Ker}_{s_{1}^{\prime}} \varphi\right) \stackrel{p_{k}(i)}{\longrightarrow} J_{k}\left(E_{1}\right) \stackrel{p_{k}(\varphi)}{\longrightarrow} J_{k}\left(E_{1}^{\prime}\right)
$$

is exact and $p_{k}(\varphi) \circ p_{k}(i)=p_{k}\left(s_{1}^{\prime}\right) \circ p_{k}\left(\pi_{1}\right)$, where $i$ is the inclusion of the fibered manifold $\operatorname{Ker}_{s_{1}^{\prime}} \varphi$ over $E$ into $E_{1}^{\prime}$.

Proof. The proposition is a consequence of Propositions 4.1 and 2.1.

If we set $E=O_{X}$ in the above proposition, then $p_{k}\left(s_{1}^{\prime}\right)=j_{k}\left(s_{1}^{\prime}\right)$ and we obtain

Corollary 4.1. If $\varphi: E \rightarrow E^{\prime}$ is a morphism of fibered manifolds of locally constant rank, then so is $p_{k}(\varphi): J_{k}(E) \rightarrow J_{k}\left(E^{\prime}\right)$. If $\varphi$ is a monomorphism (respectively an epimorphism) so is $p_{k}(\varphi)$. If $s^{\prime}$ is a section of $E^{\prime}$ over $X$ satisfying $s^{\prime}(X) \subset \varphi(E)$, then $j_{k}\left(s^{\prime}\right)(X) \subset p_{k}(\varphi)\left(J_{k}(E)\right)$ and the sequence

$$
O_{X} \longrightarrow J_{k}\left(\operatorname{Ker}_{s^{\prime}} \varphi\right) \stackrel{p_{k}(i)}{\longrightarrow} J_{k}(E) \stackrel{p_{k}(\varphi)}{\longrightarrow} J_{k}\left(E^{\prime}\right)
$$

is exact and $p_{k}(\varphi) \circ p_{k}(i)=j_{k}\left(s^{\prime}\right) \circ \pi$, whese $i$ is the inclusion of the fibered manifold $\operatorname{Ker}_{s^{\prime}} \varphi$ into $E$. 
Proposition 4.5. Let $\pi^{\prime}: E^{\prime} \rightarrow X$ be a fibered submanifold of $\pi: E \rightarrow X$ and let $\rho: M \rightarrow E$ be a fibered manifold. Then, for $k \geq 0$, considering $J_{k}\left(E^{\prime}\right)$ as a subset of $J_{k}(E)$, we have

$$
J_{k}\left(\rho^{-1}\left(E^{\prime}\right)\right)=\left(J_{k}(\rho)\right)^{-1} J_{k}\left(E^{\prime}\right)
$$

as submanifolds of $J_{k}(M)$.

Proof. This proposition follows from the implicit function theorem and Proposition 4.1.

\section{Jet bundles as affine bundles}

Let $\mathcal{O}_{x}$ denote the ring of germs $f_{x}$ at $x \in X$ of real-valued differentiable functions $f$ defined on a neighborhood of $x$ and let $\mathscr{M}_{x}$ denote its unique maximal ideal.

Lemma 5.1. Let $s$ be a section of $E$ over a neighborhood $U$ of $x_{0} \in X$ and let $f$ be any real-valued function on $U$, with $f_{x_{0}} \in \mathscr{M}_{x_{0}}^{k}$, where $k \geq 1$. Suppose that $\tilde{s}$ is any deformation of the section $s$, which is a map $\tilde{s}: U \times(-\varepsilon, \varepsilon) \rightarrow E$ satisfying the following conditions:

(i) $\tilde{s}(x, 0)=s(x)$, for all $x \in U$;

(ii) $\pi \circ \tilde{s}=p r_{1}$, where $p r_{1}$ is the projection of $U \times(-\varepsilon, \varepsilon)$ onto $U$. Then the $k$-jet at $x_{0}$ of the section

$$
x \mapsto \tilde{s}(x, f(x))
$$

of $E$ over some neighborhood of $x_{0}$ depends only on $j_{k}(s)\left(x_{0}\right)$, on the class of $f_{x_{0}}$ in $\mathscr{M}_{x_{0}}^{k} / \mathscr{M}_{x_{0}}^{k+1}$, and on the tangent vector $\left.\frac{d \tilde{s}\left(x_{0}, t\right)}{d t}\right|_{t=0} \in F_{s\left(x_{0}\right)}(E)$ to the deformation $\tilde{s}$ at $x_{0}$.

We leave the proof of this lemma to the reader. If $v_{0}=\left.\frac{d \tilde{s}\left(x_{0}, t\right)}{d t}\right|_{t=0}$ and $\bar{f}_{x_{0}}$ is the class of $f_{x_{0}}$ in $\mathscr{M}_{x_{0}}^{k} / \mathscr{M}_{x_{0}}^{k+1}$, we denote the $k$-jet at $x_{0}$ of the section (5.1) of $E$ by

$$
\left(\bar{f}_{x_{0}}, v_{0}\right)+j_{k}(s)\left(x_{0}\right) .
$$

From Lemma 5.1, it follows immediately that the $(k-1)$-jet at $x_{0}$ of the section (5.1) of $E$ is $j_{k-1}(s)\left(x_{0}\right)$ and that

$$
\left(0, v_{0}\right)+j_{k}(s)\left(x_{0}\right)=j_{k}(s)\left(x_{0}\right) .
$$

Remark 5.1. For each $\lambda \in R$, we have a deformation $\tilde{s}_{2}$ of $s$ by setting

$$
\tilde{s}_{\lambda}(x, t)=\tilde{s}(x, \lambda t), \quad \text { for }|\lambda t|<\varepsilon, x \in U,
$$

such that 


$$
\tilde{s}(x, \lambda f(x))=\tilde{s}_{2}(x, f(x))
$$

for $x$ in some neighborhood of $x_{0}$. Hence

$$
\left(\lambda \bar{f}_{x_{0}}, v_{0}\right)+j_{k}(s)\left(x_{0}\right)=\left(\bar{f}_{x_{0}}, \lambda v_{0}\right)+j_{k}(s)\left(x_{0}\right) .
$$

Remark 5.2. If $g$ is another real-valued function on $U$,

$$
\left(\bar{g}_{x_{0}}+\bar{f}_{x_{0}}, v_{0}\right)+j_{k}(s)\left(x_{0}\right)=\left(\bar{g}_{x_{0}}, v_{0}\right)+\left(\left(\bar{f}_{x_{0}}, v_{0}\right)+j_{k}(s)\left(x_{0}\right)\right) .
$$

Indeed, let

$$
\tilde{s}(x, t)=\tilde{s}(x, f(x)+t)
$$

for $x$ in some neighborhood of $x_{0}$ and $t$ in some neighborhood of $0 \in \boldsymbol{R}$. It is clear that $\tilde{\tilde{s}}$ is a deformation of the section (5.1). The tangent vector to the deformation $\tilde{\tilde{s}}$ at $x_{0}$ is $v_{0}$ because $f\left(x_{0}\right)=0$. Hence the right-hand side of (5.3) is the $k$-jet at $x_{0}$ of the section

$$
x \mapsto \tilde{s}(x, g(x))=\tilde{s}(x,(g+f)(x))
$$

of $E$ over some neighborhood of $x_{0}$, which is precisely the left-hand side of (5.3).

Remark 5.3. If $v_{0}, w_{0} \in F_{s\left(x_{0}\right)}(E)$, let $v, w$ denote vector fields tangent to the fibers of $E$ defined on some neighborhood $\bar{U}$ of $s\left(x_{0}\right)$ such that $v\left(s\left(x_{0}\right)\right)$ $=v_{0}, w\left(s\left(x_{0}\right)\right)=w_{0}$ and $[v, w] \equiv 0$ on $\bar{U}$. Then, denoting by $\exp t v, \exp t w$, $\exp t(w+v)$, the one-parameter family of (local) diffeomorphisms of $E$ generated by $v, w, w+v$ respectively, we have

$$
\exp t(w+v)=(\exp t w) \circ(\exp t v)
$$

for all sufficiently small $t$. By applying Lemma 5.1 to the deformation $\tilde{s}$ of $s$ defined by

$$
\tilde{s}(x, t)=\exp t(w+v)(s(x))=\exp t w(\exp t v(s(x)),
$$

we obtain the identity

$$
\left(\bar{f}_{x_{0}}, w_{0}+v_{0}\right)+j_{k}(s)\left(x_{0}\right)=\left(\bar{f}_{x_{0}}, w_{0}\right)+\left(\left(\bar{f}_{x_{0}}, v_{0}\right)+j_{k}(s)\left(x_{0}\right)\right)
$$

for all $v_{0}, w_{0} \in F_{s\left(x_{0}\right)}(E)$.

Remark 5.4. If $\varphi: E \rightarrow E^{\prime}$ is a morphism of fibered manifolds over $X$, then $\varphi \circ \tilde{s}$ is a deformation of the section $\varphi \circ s$ of $E^{\prime}$ over $U$. It is clear that

$$
p_{k}(\varphi)\left(\left(\bar{f}_{x_{0}}, v_{0}\right)+j_{k}(s)\left(x_{0}\right)\right)=\left(\bar{f}_{x_{0}}, \varphi_{*} v_{0}\right)+j_{k}(\varphi \circ s)\left(x_{0}\right)
$$


because $\left.\frac{d(\varphi \circ \check{s})\left(x_{0}, t\right)}{d t}\right|_{t=0}=\varphi_{*} v_{0}$ and the left-hand side of (5.5) is the $k$-jet at $x_{0}$ of the section

$$
x: \varphi(\tilde{s}(x, f(x))=(\varphi \circ \tilde{s})(x, f(x))
$$

of $E^{\prime}$ over some neighborhood of $x_{0}$.

We denote by $\pi_{0}$ and $\pi$ the natural projections of the vector bundle $S^{k} T^{*} \otimes_{E} F(E)$ onto $E$ and $X$ respectively. If $\varphi: E \rightarrow E^{\prime}$ is a morphism of fibered manifolds over $X$, then $\varphi_{*}: F(E) \rightarrow F\left(E^{\prime}\right)$ induces a morphism of vector bundles from $S^{k} T^{*} \otimes_{E} F(E)$ to $S^{k} T^{*} \otimes_{E} F\left(E^{\prime}\right)$ over $\varphi$ which we shall also denote by $\varphi_{*}$.

Proposition 5.1. There is a natural morphism of fibered manifolds over $J_{k-1}(E)$, for $k \geq 1$,

$$
S^{k} T^{*} \otimes_{E} F(E) \times_{E} J_{k}(E) \rightarrow J_{k}(E)
$$

sending $(a, p)$ into $a+p$ which induces on $J_{k}(E)$ the structure of an affine bundle over $J_{k-1}(E)$. In fact, if $\varphi: E \rightarrow E^{\prime}$ is a morphism of fibered manifolds over $X$ the following diagram is commutative:

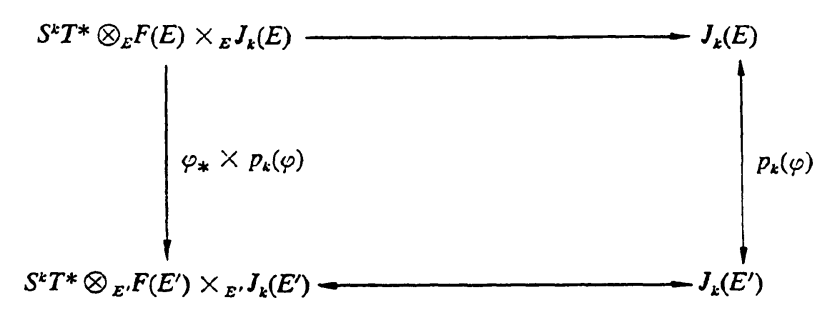

Proof. Since $\mathscr{M}_{x_{0}}^{k} / \mathscr{M}_{x_{0}}^{k+1}$ can be canonically identified with $S^{k} T_{x_{0}}^{*}$, for $x_{0} \in X$, Lemma 5.1 together with Remarks 5.1, 5.2 and 5.3 defines a mapping, for each $e \in E$, from $S^{k} T_{\pi(e)}^{*} \otimes F_{e}(E) \times J_{k}(E)_{e}$ to $J_{k}(E)_{e}$ sending $(\bar{f} \otimes v, p)$ into $\bar{f} \otimes v+p=(\bar{f}, v)+p$. This mapping determines a morphism of fibered manifolds over $J_{k-1}(E)$ from $S^{k} T^{*} \otimes{ }_{E} F(E) \times{ }_{E} J_{k}(E)$ to $J_{k}(E)$ sending $(a, p)$ into $a+p$, since the induced action of $S^{k} T^{*} \otimes_{E} F(E)$ on $J_{k}(E)$ preserves the fibers of $J_{k}(E)$ over $J_{k-1}(E)$. It is easily seen that this morphism endows $J_{k}(E)$ with the structure of an affine bundle over $J_{k-1}(E)$ modeled on the vector bundle $S^{k} T^{*} \otimes_{J_{k-1}(E)} F(E)$. The naturality of this morphism follows from Remark 5.4.

If $p_{1}, p_{2} \in J_{k}(E)$ satisfy $\pi_{k-1}\left(p_{1}\right)=\pi_{k-1}\left(p_{2}\right)$, we denote by $p_{1}-p_{2}$ the unique element of $S^{k} T^{*} \otimes_{E} F(E)$ such that

$$
\left(p_{1}-p_{2}\right)+p_{2}=p_{1} \text {. }
$$

From Proposition 5.1 and Proposition 3.7, we obtain 
Proposition 5.2. The sequence of vector bundles over $J_{k}(E)$

$$
0 \rightarrow S^{k} T^{*} \otimes_{J_{k}(E)} F(E) \stackrel{\mu}{\longrightarrow} F\left(J_{k}(E)\right) \stackrel{\pi_{k-1^{*}}}{\longrightarrow} \pi_{k-1}^{-1} F\left(J_{k-1}(E)\right) \rightarrow 0
$$

is exact.

We shall identify $S^{k} T^{*} \bigotimes_{J_{k}(E)} F(E)$ with its image in $F\left(J_{k}(E)\right)$ under the $\operatorname{map} \mu$.

Proposition 5.3. Let $\xi: W \rightarrow E$ be a vector bundle over $E$. Then:

(i) $J_{k}(\xi): J_{k}(W) \rightarrow J_{k}(E)$ is a vector bundle.

(ii) There is a natural morphism

$$
S^{k} T^{*} \otimes_{E} W \times{ }_{E} J_{k}(W) \rightarrow J_{k}(W)
$$

of fibered manifolds over $Y=J_{k}(E) \times_{J_{k-1}(E)} J_{k-1}(W)$, sending $(a, q)$ into $a+q$, induced by the morphism

$$
S^{k} T^{*} \otimes_{W} F(W) \times_{W} J_{k}(W) \rightarrow J_{k}(W)
$$

of Proposition 5.1.

(iii) There is a morphism of vector bundles over $J_{k}(E)$

$$
\varepsilon: S^{k} T^{*} \otimes_{J_{k}(E)} W \rightarrow J_{k}(W)
$$

induced by the morphism (5.6).

(iv) The sequence of vector bundles over $J_{k}(E)$

$$
0 \rightarrow S^{k} T^{*} \otimes_{J_{k}(E)} W \stackrel{\varepsilon}{\longrightarrow} J_{k}(W) \stackrel{\eta_{k-1}}{\longrightarrow} \pi_{k-1}^{-1} J_{k-1}(W) \rightarrow 0
$$

is exact, where $\eta_{l}: J_{k}(W) \rightarrow J_{l}(W)$, with $l \leq k$, and $\eta=\pi \circ \xi: W \rightarrow X$ are the natural projections.

(v) The morphism (5.6) induces on $J_{k}(W)$ the structure of an affine bundle over $Y$ modeled on the vector bundle $S^{k} T^{*} \otimes_{Y} W$.

Proof. (i) If $s_{1}, s_{2}$ are sections of $W$ over $U \subset X$, satisfying $\xi \circ s_{1}=\xi \circ s_{2}$, and $f$ is a real-valued function defined on $U$, then $s_{1}+s_{2}: x_{1} \rightarrow s_{1}(x)+s_{2}(x)$, $f \cdot s_{1}: x \mapsto f(x) s_{1}(x)$ are well-defined sections of $W$ over $U$ satisfying $\xi \circ\left(s_{1}+s_{2}\right)$ $=\xi \cdot s_{1}=\xi \circ\left(f \cdot s_{1}\right)$. Then $J_{k}(W)$ becomes a vector bundle over $J_{k}(E)$ by setting

$$
\begin{aligned}
j_{k}\left(s_{1}\right)(x)+j_{k}\left(s_{2}\right)(x) & =j_{k}\left(s_{1}+s_{2}\right)(x) \\
\lambda j_{k}\left(s_{1}\right)(x) & =j_{k}\left(\lambda \cdot s_{1}\right)(x),
\end{aligned}
$$

if $\lambda \in R, x \in U$. The zero section of this vector bundle is precisely $J_{k}(0)$, if 0 denotes the zero section of the vector bundle $W$ over $E$.

(ii) The inclusion $\mu$ of $\xi^{-1} W$ into $F(W)$ determines a map $W \times{ }_{E} J_{k}(W)$ into $F(W) \times{ }_{W} J_{k}(W)$ sending $(w, q)$ into $\left(\mu\left(\eta_{0}(q), w\right), q\right)$ and hence also a map 
from $S^{k} T^{*} \otimes_{E} W \times{ }_{E} J_{k}(W)$ to $S^{k} T^{*} \otimes_{W} F(W) \times{ }_{W} J_{k}(W)$. We define the map (5.6) to be the composition of this map from $S^{k} T^{*} \otimes_{E} W \times{ }_{E} J_{k}(W)$ to $S^{k} T^{*} \otimes_{W} F(W) \times{ }_{W} J_{k}(W)$ and of the morphism of Proposition 5.1 from $S^{k} T^{*} \otimes_{W} F(W) \times{ }_{W} J_{k}(W)$ to $J_{k}(W)$.

The morphism (5.6) can be computed explicitly in terms of the vector bundle structure on $J_{k}(W)$ as follows:

Lemma 5.2. Let $p$ be an element of $J_{k}(E)$, with $\pi_{0}(p)=e \in E$ and $\pi(p)=x_{0} \in X$, and let $w \in W$ satisfy $\xi(w)=e$. Let $f$ be any real-valued function defined on a neighborhood $U$ of $x_{0}$, with $f_{x_{0}} \in \mathscr{M}_{x_{0}}^{k}$, and $s, u$ be any sections of $W$ over $U$ satisfying $j_{k}(\xi \circ s)\left(x_{0}\right)=j_{k}(\xi \circ u)\left(x_{0}\right)=p$ and $s\left(x_{0}\right)=w$. Then

$$
\bar{f}_{x_{0}} \otimes w+j_{k}(u)\left(x_{0}\right)=j_{k}(f \cdot s+u)\left(x_{0}\right),
$$

where $\bar{f}_{x_{0}}$ is the class of $f_{x_{0}}$ in $\mathscr{M}_{x_{0}}^{k} / \mathscr{M}_{x_{0}}^{k+1}$.

Proof. The map $\tilde{u}: U \times R \rightarrow W$ defined by

$$
\tilde{u}(x, t)=u(x)+t s(x), \quad x \in U, t \in R,
$$

is a deformation of $u$, and the tangent vector at $x_{0}$ to this deformation is clearly $\mu\left(u\left(x_{0}\right), w\right) \in F_{u\left(x_{0}\right)}(W)$. Now both sides of equation (5.8) are by definition the $k$-jet at $x_{0}$ of the section

$$
x \mapsto \tilde{u}(x, f(x))=u(x)+f(x) s(x)
$$

of $W$ over $U$.

We now return to the proof of Proposition 5.3.

From Lemma 5.2, it follows directly that the map (5.6) is a morphism of fibered manifolds over $Y$.

Let $\xi: S^{k} T^{*} \otimes_{E} W \rightarrow E$ be the natural projection induced by $\xi: W \rightarrow E$. From Lemma 5.2, we obtain

Corollary 5.1. For all $a \in S^{k} T^{*} \otimes_{E} W, q_{1}, q_{2} \in J_{k}(W)$ satisfying $J_{k}(\xi)\left(q_{1}\right)$ $=J_{k}(\xi)\left(q_{2}\right)=p \in J_{k}(E)$ and $\xi(a)=\pi_{0}(p)$, we have the formulas:

$$
\begin{gathered}
a+\left(q_{1}+q_{2}\right)=\left(a+q_{1}\right)+q_{2} \\
\lambda a+\lambda q_{1}=\lambda\left(a+q_{1}\right), \quad \text { for all } \lambda \in R .
\end{gathered}
$$

(iii) Define the morphism $\varepsilon$ of fibered manifolds over $J_{k}(E)$ by

$$
\varepsilon(p, a)=a+J_{k}(0)(p),
$$

for all $p \in J_{k}(E), a \in S^{k} T^{*} \otimes_{E} W$, satisfying $\pi_{0}(p)=\xi(a)$. From (5.9), it follows that $\varepsilon$ is a morphism of vector bundles over $J_{k}(E)$.

(iv) Because $\mu: \xi^{-1} W \rightarrow F(W)$ is injective, Proposition 5.1 implies that $\varepsilon$ is injective and is therefore a monomorphism of vector bundles over $J_{k}(E)$. 
Moreover, it is clear that $\eta_{k-1} \circ \varepsilon=0$. Since $\eta_{k-1}$ is an epimorphism, counting the dimensions of the vector bundles $S^{k} T^{*} \otimes_{J_{k}(E)} W, J_{k}(W)$ and $\pi_{k-1}^{-1} J_{k-1}(W)$ over $J_{k}(E)$, we conclude that the sequence (5.7) is exact.

If $q \in J_{k}(W)$ satisfies $J_{k}(\xi)(q)=p \in J_{k}(E)$ and $\eta_{k-1}(q)=J_{k-1}(0)(p)$, we denote by $\varepsilon^{-1} q$ the unique element of $S^{k} T^{*} \otimes_{E} W$ such that

$$
\varepsilon\left(p, \varepsilon^{-1} q\right)=q,
$$

which exists and is unique by the exactness of (5.7).

(v) To verify that the morphism (5.6) induces on $J_{k}(W)$ the structure of an affine bundle over $Y$, it is sufficient to prove

Lemma 5.3. Given $q_{1}, q_{2} \in J_{k}(W)$ satisfying $J_{k}(\xi)\left(q_{1}\right)=J_{k}(\xi)\left(q_{2}\right)=p \in J_{k}(E)$ and $\eta_{k-1}\left(q_{1}\right)=\eta_{k-1}\left(q_{2}\right)$, there exists a unique element $a \in S^{k} T^{*} \otimes_{E} W$ such that

$$
a+q_{2}=q_{1} .
$$

In fact, $a$ is the unique element $\varepsilon^{-1}\left(q_{1}-q_{2}\right)$ of $S^{k} T^{*} \otimes_{E} W$ which satisfies

$$
\varepsilon\left(p, \varepsilon^{-1}\left(q_{1}-q_{2}\right)\right)=q_{1}-q_{2},
$$

where $q_{1}-q_{2}$ is the element of $J_{k}(W)$ determined by the vector bundle structure of $J_{k}(E)$.

Proof. Equation (5.10) is equivalent to

$$
\varepsilon^{-1}\left(q_{1}-q_{2}\right)+q_{2}=q_{1} .
$$

Indeed, we can rewrite $(5.10)$ as

$$
\varepsilon^{-1}\left(q_{1}-q_{2}\right)+J_{k}(0)(p)=q_{1}-q_{2} .
$$

The above equation is equivalent to

$$
\left(\varepsilon^{-1}\left(q_{1}-q_{2}\right)+J_{k}(0)(p)\right)+q_{2}=q_{1},
$$

which, by Corollary 5.1 , is the same as

$$
\varepsilon^{-1}\left(q_{1}-q_{2}\right)+\left(J_{k}(0)(p)+q_{2}\right)=q_{1}
$$

or equation (5.11).

If we set $E=O_{X}$ in the above proposition, we obtain the exact sequence of vector bundles over $X$

$$
0 \rightarrow S^{k} T^{*} \otimes W \stackrel{\varepsilon}{\longrightarrow} J_{k}(W) \stackrel{\xi_{k-1}}{\longrightarrow} J_{k-1}(W) \rightarrow 0
$$

for any vector bundle $\xi: W \rightarrow X$ over $X$.

Proposition 5.4. If $\varphi: E \rightarrow E^{\prime}$ is a morphism of fibered manifolds over $X$, then, for $k \geq 1$, the map $p_{k}(\varphi): J_{k}(E) \rightarrow J_{k}\left(E^{\prime}\right)$ is a morphism of affine 
bundles over $p_{k-1}(\varphi)$ whose associated morphism of vector bundles is $\varphi_{*}: S^{k} T^{*} \otimes_{J_{\boldsymbol{k}}(E)} F(E) \rightarrow S^{k} T^{*} \otimes_{J_{k}\left(E^{\prime}\right)} F\left(E^{\prime}\right)$.

This proposition is a direct consequence of Proposition 5.1.

Proposition 5.5. For $l \geq 1$, the map $p_{l}\left(\mathrm{id}_{k}\right): J_{k+l}(E) \rightarrow J_{l}\left(J_{k}(E)\right)$ is a monomorphism of affine bundles over $p_{l-1}\left(\mathrm{id}_{k}\right): J_{k+l-1}(E) \rightarrow J_{l-1}\left(J_{k}(E)\right)$.

Proof. The diagram

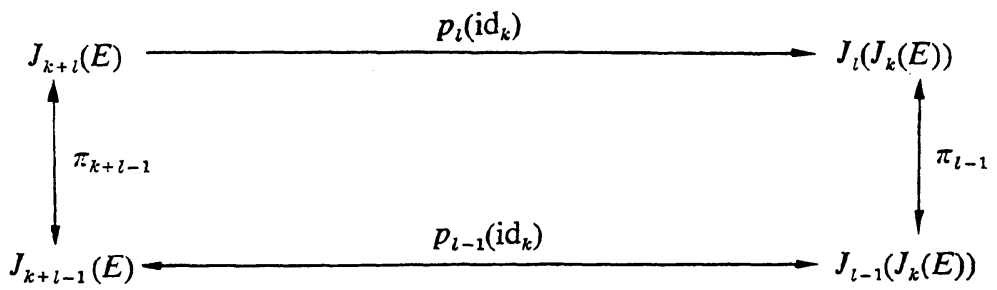

is easily seen to commute, so that $p_{l}\left(\mathrm{id}_{k}\right)$ is a morphism of fibered manifolds over $p_{l-1}\left(\mathrm{id}_{k}\right)$ which is easily seen to be a monomorphism of affine bundles.

Define a monomorphism $\Delta_{l, k}: S^{k+l} T^{*} \rightarrow S^{\imath} T^{*} \otimes S^{k} T^{*}$ of vector bundles over $X$ as follows: let $\Delta_{l, k}$ be the composition of the natural inclusion of $S^{k+l} T^{*}$ into $\otimes^{k+l} T^{*}=\otimes^{l} T^{*} \otimes \otimes^{k} T^{*}$ and the map from $\otimes^{l} T^{*} \otimes \otimes^{k} T^{*}$ to $S^{l} T^{*} \otimes S^{k} T^{*}$ induced by the natural projections of $\otimes^{l} T^{*}, \otimes^{k} T^{*}$ onto $S^{l} T^{*}, S^{k} T^{*}$. If $l=1$, we set $\delta=\delta_{k}=\Delta_{1, k}$.

By Proposition 4.3, the following diagram commutes:

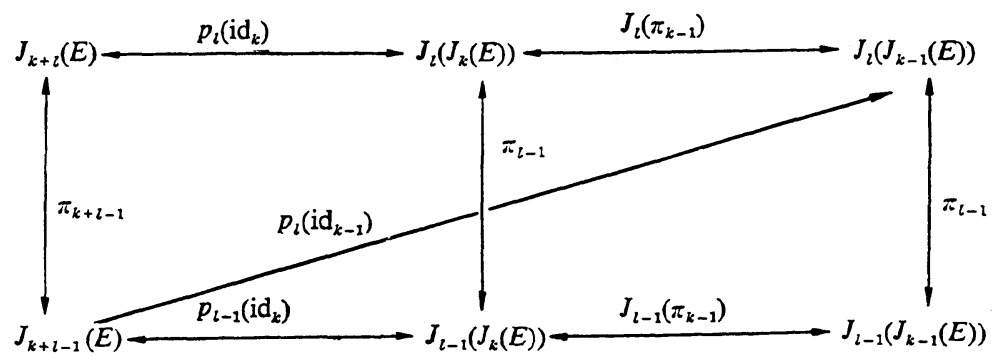

By Propositions 5.4. and 5.5, the composition $J_{l}\left(\pi_{k-1}\right) \circ p_{l}\left(\mathrm{id}_{k}\right)$ is a morphism of affine bundles over $J_{l-1}\left(\pi_{k-1}\right) \circ p_{l-1}\left(\mathrm{id}_{k}\right)$ whose associated morphism of vector bundles is the composition

$$
\begin{aligned}
S^{k+l} T^{*} \otimes_{J_{k+l-1}(E)} F(E) \rightarrow S^{\imath} T^{*} & \bigotimes_{J_{l-1}\left(J_{k}(E)\right)} F\left(J_{k}(E)\right) \\
& \stackrel{\pi_{k-1^{*}}}{\longrightarrow} S^{\imath} T^{*} \bigotimes_{J_{l-1}\left(J_{k-1}(E)\right)} F\left(J_{k-1}(E)\right)
\end{aligned}
$$

where the map from $S^{k+l} T^{*} \otimes_{J_{k+l-1}(E)} F(E)$ to $S^{l} T^{*} \otimes_{J_{l-1}\left(J_{k}(E)\right)} F\left(J_{k}(E)\right)$ is the morphism of vector bundles associated to $p_{l}\left(\mathrm{id}_{k}\right)$. Since the morphism $J_{l}\left(\pi_{k-1}\right) \circ p_{l}\left(\mathrm{id}_{k}\right)$ factors through $J_{k+l-1}(E)$, its associated morphism of vector bundles is the zero morphism over $J_{l-1}\left(\pi_{k-1}\right) \circ p_{l-1}\left(\mathrm{id}_{k}\right)$. Therefore, by 
Proposition 5.2, the morphism of vector bundles associated to $p_{l}\left(\mathrm{id}_{k}\right)$ is determined by a morphism of vector bundles from $S^{k+l} T^{*} \otimes_{J_{k+l-1}(E)} F(E)$ to $S^{l} T^{*} \otimes S^{k} T^{*} \otimes_{J_{l-1}\left(J_{k}(E)\right)} F(E)$ over $p_{l-1}\left(\mathrm{id}_{k}\right)$, which is easily seen to be precisely the morphism induced by $\Delta_{l, k}$.

Proposition 5.6. If $\varphi: J_{k}(E) \rightarrow E^{\prime}$ is a morphism of fibered manifolds over $X$, then, for $l \geq 1$, the map $p_{l}(\varphi): J_{k+l}(E) \rightarrow J_{l}\left(E^{\prime}\right)$ is a morphism of affine bundles over $p_{l-1}(\varphi)$, whose associated morphism of vector bundles is induced by the map

$$
\varphi_{*} \circ \Delta_{l, k}: S^{k+l} T^{*} \otimes_{J_{k}(E)} F(E) \rightarrow S^{l} T^{*} \otimes_{E^{\prime}} F\left(E^{\prime}\right)
$$

over $\varphi$.

Proof. The diagram

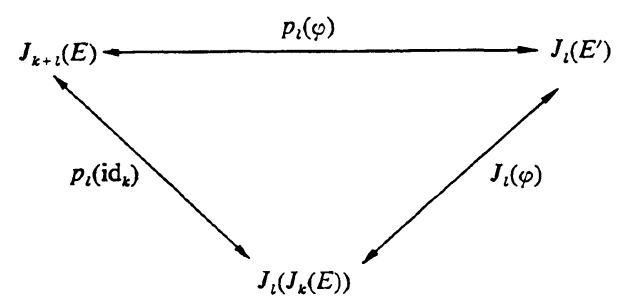

commutes by Proposition 4.3. Hence by Propositions 5.4 and $5.5, p_{l}(\varphi)$ is a morphism of affine bundles whose associated morphism of vector bundles is the map

$$
\varphi_{*} \circ \Delta_{l, k}: S^{k+\imath} T^{*} \otimes_{J_{k+l-1}(E)} F(E) \rightarrow S^{l} T^{*} \otimes_{J_{l-1}\left(E^{\prime}\right)} F\left(E^{\prime}\right)
$$

over $p_{l-1}(\varphi)$.

If $\varphi$ is a morphism of an open fibered submanifold $F$ of $J_{k}(E)$ into $E^{\prime}$, analogues of Propositions 5.4, 5.6 hold for $\varphi$.

We now conclude this section by giving an alternate description of the affine bundle structure which $J_{k}(E)$ possesses.

Let $p$ be an element of $J_{k}(E)$ and let $s$ be a section of $E$ over a neighborhood of $x=\pi(p)$ such that $j_{k}(s)(x)=p$; set $q=\pi_{k-1}(p)$. Then the linear map $j_{k-1}(s)_{*}: T_{x} \rightarrow T q\left(J_{k-1}(E)\right)$ depends only on $p$ and will be denoted simply by $p_{*}$. In fact, $p_{*}$ determines $p$ uniquely; indeed, if $p^{\prime}$ is an element of $J_{k}(E)$ such that $\pi_{k-1}\left(p^{\prime}\right)=q$ and $p_{*}^{\prime}=p_{*}$ as maps from $T_{x}$ to $T q\left(J_{k-1}(E)\right)$, then $p^{\prime}$ $=p$. In general, if $p^{\prime} \in J_{k}(E)$ satisfies $\pi_{k-1}\left(p^{\prime}\right)=q$, then $\pi_{k-1 *} p_{*}=\pi_{k-1_{*}} p_{*}^{\prime}$ and so $p_{*}^{\prime}-p_{*}$ belongs to $T^{*} \otimes S^{k-1} T^{*} \otimes_{J_{k-1}(E)} F(E)$ by Proposition 5.2. The map from $J_{k}(E) q$ to $\left(T^{*} \otimes S^{k-1} T^{*} \otimes_{J_{k-1}(E)} F(E)\right) q$ sending $p^{\prime}$ into $p_{*}^{\prime}-p_{*}$ is injective. Actually $p_{*}^{\prime}-p_{*}$ belongs to the subspace $\left(\delta\left(S^{k} T^{*}\right) \otimes_{J_{k-1}(E)} F(E)\right) q$ of $\left(T^{*} \otimes S^{k-1} T^{*} \otimes{ }_{J_{k-1}(E)} F(E)\right) q$ and every element of this subspace is of the form $p_{*}^{\prime}-p_{*}$, for some $p^{\prime} \in J_{k}(E) q$. Since $\delta$ is injective, we can identify $S^{k} T^{*}$ 
with its image in $T^{*} \otimes S^{k-1} T^{*}$ under the map $\delta$. Hence $p$ determines a bijective map from $J_{k}(E) q$ to $\left(S^{k} T^{*} \otimes_{J_{k-1}(E)} F(E)\right) q$. It is easily seen that this map endows $J_{k}(E) q$ with the structure of an affine space modeled on the vector space $\left(S^{k} T^{*} \otimes_{J_{k-1}(E)} F(E)\right) q$. This gives rise to a morphism of fibered manifolds over $J_{k-1}(E)$

$$
S^{k} T^{*} \otimes_{E} F(E) \times{ }_{E} J_{k}(E) \rightarrow J_{k}(E)
$$

sending $(a, p)$ into the unique element $p^{\prime}$ of $J_{k}(E)$ such that $p_{*}^{\prime}=\delta\left(\pi_{k-1}(p), a\right)$ $+p_{*}$. It is readily verified that this morphism is precisely the morphism of Proposition 5.1.

\section{The Spencer cohomology}

We shall henceforth assume that all the components of the differentiable manifold $X$ have the same dimension $n$.

We have a morphism of vector bundles over $X$

$$
\delta: S^{k} T^{*} \longrightarrow T^{*} \otimes S^{k-1} T^{*}
$$

defined in $\S 5$. We extend $\delta$ to a vector bundle morphism

$$
\delta: \wedge^{j} T^{*} \otimes S^{k} T^{*} \longrightarrow \wedge^{j+1} T^{*} \otimes S^{k-1} T^{*}
$$

sending $\omega \otimes u$ into $(-1)^{j} \omega \wedge \delta u$, where $\omega \in \wedge^{j} T^{*}, u \in S^{k} T^{*}$. Then we have the complex

$$
\begin{aligned}
0 \longrightarrow S^{k} T^{*} \stackrel{\delta}{\longrightarrow} T^{*} \otimes S^{k-1} T^{*} & \stackrel{\delta}{\longrightarrow} \wedge^{2} T^{*} \otimes S^{k-2} T^{*} \stackrel{\delta}{\longrightarrow} \cdots \\
\longrightarrow & \wedge^{n} T^{*} \otimes S^{k-n} T^{*} \longrightarrow 0
\end{aligned}
$$

(i.e., $\delta^{2}=0$ ), where $S^{\imath} T^{*}=0$ for $l<0$. We call $\delta$ the formal differentiation operator and we now state the formal Poincaré lemma:

Lemma 6.1 (see D. C. Spencer [8], D. G. Quillen [7], or S. Sternberg [9]). The sequences (6.1) are exact for $k \geq 1$.

Let $Y$ be a differentiable manifold and let $f: Y \rightarrow X$ be a differentiable map. Let $W$ be a vector bundle over $Y$. Suppose that $g_{k}$ is a family of subspaces of $S^{k} T^{*} \otimes_{Y} W$ over $Y$, where $k \geq 1$. Define the $l$-th prolongation $g_{k+l}$ of $g_{k}$ to be the kernel of the composition $\phi_{l}$ :

$$
S^{k+l} T^{*} \otimes_{Y} W \stackrel{\Delta_{l, k}}{\longrightarrow} S^{l} T^{*} \otimes S^{k} T^{*} \otimes_{Y} W \stackrel{\varphi}{\longrightarrow} S^{l} T^{*} \otimes_{Y}\left(\left(S^{k} T^{*} \otimes_{Y} W\right) / g_{k}\right)
$$

where the map $\psi$ is induced by the natural projection of $S^{k} T^{*} \otimes_{Y} W$ onto $\left(S^{k} T^{*} \otimes_{Y} W\right) / g_{k}$. We set $g_{k-l}=S^{k-l} T^{*} \otimes_{Y} W$ for $l<0$.

Since the diagram 


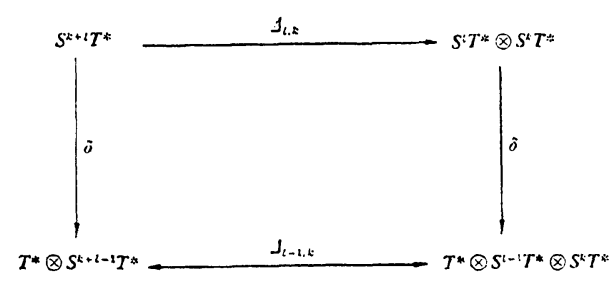

commutes, it is easily seen that $\delta\left(g_{k+l}\right) \subset T^{*} \otimes_{Y} g_{k+l-1}$ and hence $\delta$ induces a map

$$
\delta: \wedge^{j} T^{*} \otimes_{Y} g_{k+l} \longrightarrow \wedge^{j+1} T^{*} \otimes_{Y} g_{k+l-1} .
$$

Definition 6.1. The Spencer cohomology of $g_{k}$ is the cohomology of the sequences

$$
\begin{aligned}
0 \longrightarrow & g_{m} \stackrel{\delta}{\longrightarrow} T^{*} \otimes_{Y} g_{m-1} \stackrel{\delta}{\longrightarrow} \wedge^{2} T^{*} \otimes_{Y} g_{m-2} \stackrel{\delta}{\longrightarrow} \cdots \\
& \longrightarrow \wedge^{m-k} T^{*} \otimes_{Y} g_{k} \stackrel{\delta}{\longrightarrow} \wedge^{m-k+1} T^{*} \otimes S^{k-1} T^{*} \otimes_{Y} W
\end{aligned}
$$

where $m \geq k$. We denote by $H^{m-j, j}=H^{m-j, j}\left(g_{k}\right)$ the cohomology of the sequence (6.3) at $\wedge^{j} T^{*} \otimes_{Y} g_{m-j}$. We say that $g_{k}$ is involutive if the sequences (6.3) are exact and that $g_{k}$ is $r$-acyclic if $H^{m, j}=0$ for $m \geq k, 0 \leq j \leq r$.

Lemma 6.2. The sequences

$$
0 \longrightarrow g_{k+l} \stackrel{\delta}{\longrightarrow} T^{*} \otimes_{Y} g_{k+l-1} \stackrel{\delta}{\longrightarrow} \wedge^{2} T^{*} \otimes_{Y} g_{k+l-2}
$$

are exact for $l \geq 1$, that is, $g_{k}$ is 1 -acyclic.

Proof. The commutativity of diagram (6.2) implies the commutativity of the following diagram, whose rows are exact, where $l \geq 1$ :

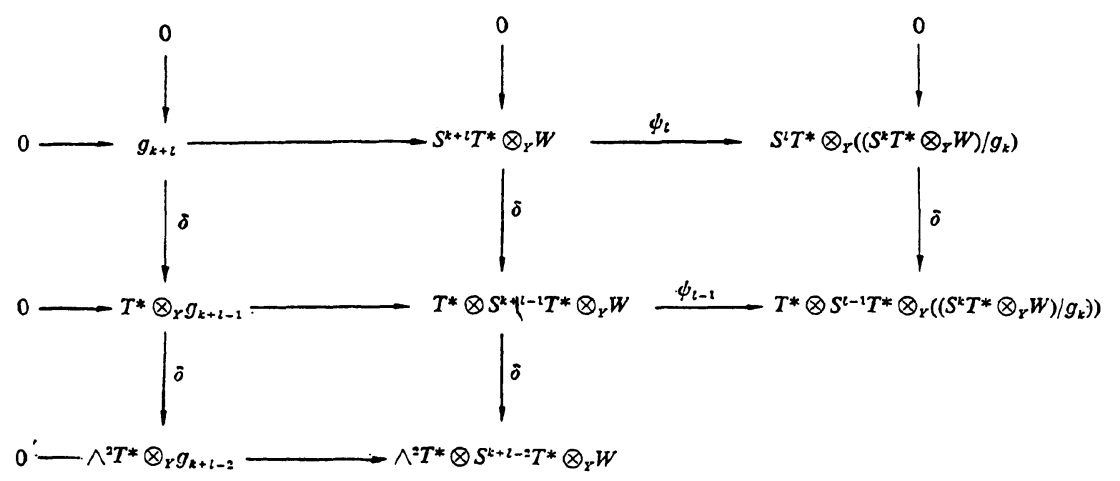

By Lemma 6.1, the last two columns of this diagram are also exact and hence so is the first.

Lemma 6.3. The m-th prolongation $g_{(k+l)+m}$ of the family of subspaces: $g_{k+l} \subset S^{k+\imath} T^{*} \otimes_{Y} W$ is the same as the $(l+m)$-th prolongation of $g_{k}$. 
Proof. We proceed by induction on $m$. For $m=0$, the lemma is trivial. Assume that $m \geq 1$ and that $g_{(k+l)+q}=g_{k+l+q}$ for $q<m$. By Lemma 6.2, the diagram

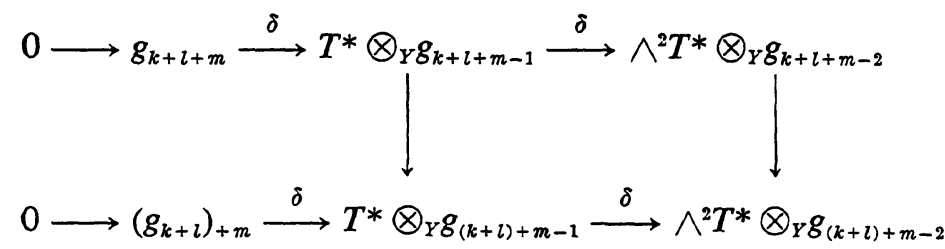

is exact and commutative, so that $g_{(k+l)+m}=g_{k+l+m}$.

We now state the $\delta$-Poincaré lemma (see D. G. Quillen [7] or S. Sternberg [9]).

Lemma 6.4. If the dimension of $W_{y}$ is independent of $y \in Y$, there exists an integer $k_{0}>k$ depending only on $n, k$ and the dimension of $W_{y}, y \in Y$, such that $g_{k_{0}}$ is involutive.

Hence, by Lemma 6.3 , the Spencer cohomology groups $H^{m, j}\left(g_{k}\right)$ vanish for $m \geq k_{0}$.

Lemma 6.5. Let $W^{\prime}$ be a vector bundle over $Y$ and let $g_{k}$ be the kernel of a morphism of vector bundles $\psi: S^{k} T^{*} \otimes_{Y} W \rightarrow W^{\prime}$ over $Y$. If $g_{k}$ is 2-acyclic and if $g_{k+1}$ is a vector bundle over $Y$, then $g_{k+l}$ is a vector bundle over $Y$ for $l \geq 1$.

Proof. The exactness of the sequence

$$
T^{*} \otimes_{Y} g_{k+1} \stackrel{\delta}{\longrightarrow} \wedge^{2} T^{*} \otimes_{Y} g_{k} \stackrel{\delta}{\longrightarrow} \wedge^{3} T^{*} \otimes S^{k-1} T^{*} \otimes_{Y} W
$$

implies the exactness of the sequence

$$
\begin{aligned}
& 0 \longrightarrow \delta\left(T^{*} \otimes_{Y} g_{k+1}\right) \longrightarrow \wedge^{2} T^{*} \otimes S^{k} T^{*} \otimes_{Y} W \\
& \stackrel{\psi \oplus \delta}{\longrightarrow}\left(\wedge^{2} T^{*} \otimes_{Y} W^{\prime}\right) \oplus\left(\wedge^{3} T^{*} \otimes S^{k-1} T^{*} \otimes_{Y} W\right)
\end{aligned}
$$

where $\psi \oplus \delta$ is the morphism of vector bundles over $Y$ sending $u$ into $\phi(u) \oplus \delta u$. Hence the function $y \mapsto \operatorname{dim}\left(\delta\left(T^{*} \otimes_{Y} g_{k+1}\right)\right)_{y}$ on $Y$ is upper semi-continuous. Since $g_{k+2}$ is the kernel of the composition

$$
S^{k+2} T^{*} \otimes_{Y} W \stackrel{\Delta_{2, k}}{\longrightarrow} S^{2} T^{*} \otimes S^{k} T^{*} \otimes_{Y} W \stackrel{\phi}{\longrightarrow} S^{2} T^{*} \otimes_{Y} W^{\prime},
$$

the function $y_{\longmapsto} \mapsto \operatorname{dim}\left(g_{k+2}\right)_{y}$ on $Y$ is also upper semi-continuous. The sequence

$$
0 \longrightarrow g_{k+2} \stackrel{\delta}{\longrightarrow} T^{*} \otimes_{Y} g_{k+1} \stackrel{\delta}{\longrightarrow} \delta\left(T^{*} \otimes_{Y} g_{k+1}\right) \longrightarrow 0
$$

is exact by Lemma 6.2. Therefore, taking the Euler-Poincaré characteristic of (6.4), we see that the function $y_{\mapsto} \rightarrow \operatorname{dim}\left(g_{k+2}\right)_{y}+\operatorname{dim}\left(\delta\left(T^{*} \otimes_{Y} g_{k+1}\right)\right)_{y}$ on $Y$ is locally constant, because $g_{k+1}$ is a vector bundle over $Y$. Hence $g_{k+2}$ and $\delta\left(T^{*} \otimes_{Y} g_{k+1}\right)$ are both vector bundles. Consider the exact sequence, for $l \geq 0$, 
$0 \rightarrow g_{k+l+3} \stackrel{\delta}{\longrightarrow} T^{*} \otimes_{Y} g_{k+l+2} \stackrel{\delta}{\longrightarrow} \wedge^{2} T^{*} \otimes_{Y} g_{k+l+1} \stackrel{\delta}{\longrightarrow} \wedge^{3} T^{*} \otimes S^{k+l} T^{*} \otimes_{Y} W$.

Lemma 3.3 of [5] implies that $g_{k+l+3}$ is a vector bundle whenever $g_{k+l+2}$ and $g_{k+l+1}$ are vector bundles, completing the proof.

\section{Differential equations}

Definition 7.1. A partial differential equation $R_{k}$ of order $k$ on $E$ is a fibered submanifold of $\pi: J_{k}(E) \rightarrow X$. A solution of $R_{k}$ is a section $s$ of $E$ over an open set $U \subset X$ such that $j_{k}(s)(x) \in R_{k}$ for all $x \in U$. The $l$-th prolongation of $R_{k}$ is the subset

$$
R_{k+l}=J_{l}\left(R_{k}\right) \cap J_{k+l}(E)
$$

of $J_{k+l}(E)$, where $J_{l}\left(R_{k}\right)$ is considered as a subset of $J_{l}\left(J_{k}(E)\right)$. The symbol of $R_{k}$ is the family of subspaces

$$
g_{k}=F\left(R_{k}\right) \cap\left\{S^{k} T^{*} \otimes_{R_{k}} F(E)\right\}
$$

of the vector bundle $S^{k} T^{*} \otimes_{R_{k}} F(E)$. Note that $g_{k}$ is the kernel of the morphism of vector bundles over $R_{k}$

$$
S^{k} T^{*} \otimes_{R_{k}} F(E) \rightarrow\left(F\left(J_{k}(E)\right) \mid R_{k}\right) / F\left(R_{k}\right) .
$$

Note that $\pi_{k+l}\left(R_{k+l+1}\right) \subset R_{k+l}$. Let $g_{k+l}$ be the l-th prolongation of $g_{k}$; then $g_{k+l}$ is a sub-family of vector spaces of the vector bundle $S^{k+l} T^{*} \otimes_{R_{k}} F(E)$.

If $F$ is an open fibered submanifold of $J_{k}(E)$ over $X$, by Proposition 2.1, any morphism $\varphi: F \rightarrow E^{\prime}$ of fibered manifolds of locally constant rank and a section $s^{\prime}$ of $E^{\prime}$ over $X$ satisfying $s^{\prime}(X) \subset \varphi(F)$ determine a partial differential equation $R_{k}=\operatorname{Ker}_{s^{\prime}} \varphi$. For such an equation, we have

$$
R_{k+l}=\operatorname{Ker}_{j_{l}\left(s^{\prime}\right)} p_{l}(\varphi) \text {. }
$$

In fact, the morphism $p_{l}\left(\mathrm{id}_{k}\right): J_{k+l}(E) \rightarrow J_{l}\left(J_{k}(E)\right)$ induces a morphism $p_{l}\left(\mathrm{id}_{k}\right)$ from the open fibered submanifold $\pi_{k}^{-1} F$ of $J_{k+l}(E)$ into $J_{l}(F)$; the diagram

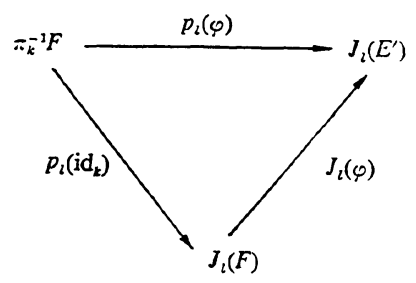

commutes by Proposition 4.3 and so by Corollary 4.1 , we conclude that 


$$
R_{k+l}=\left(\operatorname{Ker}_{j_{l}\left(s^{\prime}\right)} J_{l}(\varphi)\right) \cap J_{k+l}(E)=\operatorname{Ker}_{j_{l}\left(s^{\prime}\right)} p_{l}(\varphi)
$$

Moreover by Proposition 2.1, for $p \in R_{k}$ the vector space $\left(g_{k}\right)_{p}$ is the kernel of the map $\varphi_{*}:\left(S^{k} T^{*} \otimes_{R_{k}} F(E)\right)_{p} \rightarrow F_{\varphi(p)}\left(E^{\prime}\right)$.

Conversely, given an equation $R_{k} \subset J_{k}(E)$ of order $k$ on $E$, such fibered manifolds $F, E^{\prime}$, morphism $\varphi$ and section $s^{\prime}$ of $E^{\prime}$ such that $R_{k}=\operatorname{Ker}_{s^{\prime}} \varphi$ exist if and only if the normal bundle of the imbedding of $R_{k}$ into $J_{k}(E)$ is isomorphic to a vector bundle induced by $\pi$ from a vector bundle over $X$, by Proposition 2.2. Hence the condition that a fibered submanifold $R_{k}$ of $J_{k}(E)$ be the kernel of a morphism $\varphi: J_{k}(E) \rightarrow E^{\prime}$ of locally constant rank imposes a topological restriction on $R_{k}$. For this reason, we have given the above definition of a partial differential equation rather than defining an equation as a kernel of such a morphism $\varphi$. In the linear case, both points of view coincide (see [5]).

Definition 7.2. We say that a partial differential equation $R_{k} \subset J_{k}(E)$ of order $k$ on $E$ is formally integrable if, for $l \geq 0, g_{k+l+1}$ is a vector bundle over $R_{k}$, and the map $\pi_{k+l}: R_{k+l+1} \rightarrow R_{k+l}$ is surjective.

Proposition 7.1. Let $R_{k} \subset J_{k}(E)$ be a partial differential equation of order $k$ on $E$. Then the following statements are equivalent:

(i) $g_{k+1}$ is a vector bundle over $R_{k}$ and the map $\pi_{k}: R_{k+1} \rightarrow R_{k}$ is surjective.

(ii) $\pi_{k}: R_{k+1} \rightarrow R_{k}$ is a fibered submanifold of $\pi_{k}: J_{k+1}(E) \mid R_{k} \rightarrow R_{k}$.

(iii) $\pi_{k}: R_{k+1} \rightarrow R_{k}$ is an affine sub-bundle of $\pi_{k}: J_{k+1}(E) \mid R_{k} \rightarrow R_{k}$ modeled on the vector bundle $g_{k+1}$.

Moreover, if any one of these assertions is satisfied, the l-th prolongation $R_{(k+1)+l}$ of the equation $R_{k+1}$ is the same as the $(l+1)$-th prolongation of the equation $R_{k}$.

Proof. By Lemma 2.1, for each $p \in R_{k}$, there are an open neighborhood $V$ of $p$ in $J_{k}(E)$, a fibered manifold $E^{\prime}$ over $U=\pi V$, a section $s^{\prime}$ of $E^{\prime}$ over $U$ and a morphism $\varphi: V \rightarrow E^{\prime}$ of fibered manifolds of constant rank such that $R_{k} \cap V=\operatorname{Ker}_{s^{\prime}} \varphi$. Then, by Proposition 5.6, $p_{l}(\varphi): J_{k+l}(E) \mid V \rightarrow J_{l}\left(E^{\prime}\right)$ is a morphism of affine bundles over $p_{l-1}(\varphi): J_{k+l-1}(E) \mid V \rightarrow J_{l-1}\left(E^{\prime}\right)$ whose associated morphism of vector bundles is induced by the map $\varphi_{*} \circ \Delta_{l, k}$ over $\varphi$. Since $\varphi$ has constant rank, Proposition 4.3 and Corollary 4.1 imply, by a previous argument, that $R_{k+l} \cap \pi_{k}^{-1}(V)=\operatorname{Ker}_{j_{l}\left(s^{\prime}\right)} p_{l}(\varphi)$; Proposition 2.1 implies that $\left(g_{k+l}\right)_{p}$ is the kernel of $\varphi_{*} \circ \Delta_{l, k}:\left(S^{k+l} T^{*} \otimes_{R_{k}} F(E)\right)_{p} \rightarrow\left(S^{l} T^{*} \otimes_{E^{\prime}} F\left(E^{\prime}\right)\right)_{\varphi(p)}$, if $p \in R_{k} \cap V$.

We first show that (i) implies (iii). Since $g_{k+1}$ is a vector bundle over $R_{k}$, by Proposition 3.3, it follows that $p_{1}(\varphi) \mid \pi_{k}^{-1}\left(R_{k} \cap V\right)$ has locally constant rank and that (iii) holds.

Clearly (iii) implies (ii). Finally, let us prove that (ii) implies (i). To verify that $g_{k+1}$ is a vector bundle over $R_{k}$, it suffices to show that we have an exact sequence 


$$
0 \longrightarrow\left(g_{k+1}\right) p \longrightarrow F_{q}\left(R_{k+1}\right) \stackrel{\pi_{k^{*}}}{\longrightarrow} F p\left(R_{k}\right) \longrightarrow 0
$$

for all $q \in R_{k+1}, p \in R_{k}$, with $\pi_{k}(q)=p$. The map

$$
S^{k+1} T^{*} \otimes_{E} F(E) \times_{E} J_{k+1}(E) \rightarrow J_{k+1}(E)
$$

of Proposition 5.1 induces a map

$$
g_{k+1} \times_{R_{k}} R_{k+1} \rightarrow J_{k+1}(E)
$$

whose image is $R_{k+1}$. Indeed, the image of this map is contained in $J_{1}\left(R_{k}\right)$, since $g_{k+1}=\left(S^{k+1} T^{*} \bigotimes_{R_{k}} F(E)\right) \cap\left(T^{*} \bigotimes_{R_{k}} F\left(R_{k}\right)\right)$ and $p_{1}\left(\mathrm{id}_{k}\right): J_{k+1}(E) \rightarrow J_{1}\left(J_{k}(E)\right)$ is a monomorphism of affine bundles over $J_{k}(E)$. Given $q_{1}, q_{2} \in R_{k+1}$, with $\pi_{k}\left(q_{1}\right)$ $=\pi_{k}\left(q_{2}\right)$, by Proposition 5.1 there exist elements $a \in T^{*} \bigotimes_{R_{k}} F\left(R_{k}\right), a^{\prime} \in S^{k+1} T^{*}$ $\otimes_{E} F(E)$ such that $a+q_{1}=q_{2}, a^{\prime}+q_{1}=q_{2}$ as elements of $J_{1}\left(R_{k}\right)$ and $J_{k+1}(E)$ respectively. Since $p_{1}\left(\mathrm{id}_{k}\right): J_{k+1}(E) \rightarrow J_{1}\left(J_{k}(E)\right)$ and the mapping of $J_{1}\left(R_{k}\right)$ into $J_{1}\left(J_{k}(E)\right)$ are both monomorphisms of affine bundles, we have $a=a^{\prime} \in g_{k+1}$. We have thus shown that, for all $p \in R_{k}$, the fiber $\left(R_{k+1}\right)_{p}$ is an affine subspace of $\left(J_{k+1}(E)\right)_{p}$ modeled on $\left(g_{k+1}\right)_{p}$. By Proposition 3.7, we have a natural inclusion of $\left(g_{k+1}\right) p$ into $F_{q}\left(R_{k+1}\right)$ for all $q \in R_{k+1}$, with $\pi_{k}(q)=p$, determining the sequence (7.1). Since $F_{q}\left(R_{k+1}\right) \subset F_{q}\left(J_{1}\left(R_{k}\right)\right) \cap F_{q}\left(J_{k+1}(E)\right)$, this sequence is exact.

We now assume that (i), (ii) or (iii) holds. To prove the remaining part of the proposition, it suffices to show that

$$
R_{k+l+1} \cap \pi_{k}^{-1}(V)=R_{(k+1)+l} \cap \pi_{k}^{-1}(V),
$$

where $\pi_{k}^{-1}(V)$ denotes, as throughout the rest of this proof, the inverse image in $J_{k+l+1}(E)$ of the open set $V \subset J_{k}(E)$ under the map $\pi_{k}$. We set $V^{\prime}=R_{k} \cap V$ and $\rho=\pi_{k}: J_{k+1}(E) \rightarrow J_{k}(E)$. First note that $V^{\prime}$ is a fibered submanifold of $V$ over $U$ and that, since $\rho: R_{k+1} \rightarrow R_{k}$ is a fibered manifold, $R_{k+1} \cap \rho^{-1}\left(V^{\prime}\right)$ $=R_{k+1} \cap \rho^{-1}(V)$ is a fibered manifold over $U$. The sequences of fibered manifolds over $U$

$$
\begin{gathered}
O_{U} \longrightarrow V^{\prime} \longrightarrow V \stackrel{\varphi}{\longrightarrow} E^{\prime} \\
O_{U} \longrightarrow R_{k+1} \cap \rho^{-1}(V) \longrightarrow \rho^{-1}\left(V^{\prime}\right) \stackrel{p_{1}(\varphi)}{\longrightarrow} J_{1}\left(E^{\prime}\right)
\end{gathered}
$$

are exact, since $\varphi$ and the restriction of $p_{1}(\varphi)$ to $\rho^{-1}\left(V^{\prime}\right)$ have locally constant rank. Hence, by Corollary 4.1 , the sequences of fibered manifolds over $U$

$$
\begin{gathered}
O_{U} \longrightarrow J_{l}\left(V^{\prime}\right) \longrightarrow J_{l}(V) \stackrel{J_{l}(\varphi)}{\longrightarrow} J_{l}\left(E^{\prime}\right) \\
O_{U} \longrightarrow J_{l}\left(R_{k+1} \cap \rho^{-1}(V)\right) \longrightarrow J_{l}\left(\rho^{-1}\left(V^{\prime}\right)\right) \stackrel{J_{l}\left(p_{1}(\varphi)\right)}{\longrightarrow} J_{l}\left(J_{1}\left(E^{\prime}\right)\right)
\end{gathered}
$$


are also exact. Since $\rho^{-1}(V)$ is open in $J_{k+1}(E)$, we have

$$
\begin{aligned}
R_{(k+1)+l} \cap \pi_{k}^{-1}(V) & =J_{l}\left(R_{k+1}\right) \cap \pi_{k}^{-1}(V) \\
& =J_{l}\left(R_{k+1} \cap \rho^{-1}(V)\right) \cap \pi_{k}^{-1}(V) .
\end{aligned}
$$

The exactness of (7.3) and Proposition 4.5 imply that

$$
\begin{aligned}
R_{(k+1)+2} \cap \pi_{k}^{-1}(V) & =\left\{\operatorname{Ker}_{j_{l}\left(j_{1}\left(s^{\prime}\right)\right)} J_{l}\left(p_{1}(\varphi)\right) \mid J_{l}\left(\rho^{-1}\left(V^{\prime}\right)\right)\right\} \cap \pi_{k}^{-1}(V) \\
& =\left\{\operatorname{Ker}_{j_{l}\left(j_{1}\left(s^{\prime}\right)\right)} J_{l}\left(p_{1}(\varphi)\right) \mid\left(J_{l}(\rho)\right)^{-1} J_{l}\left(V^{\prime}\right)\right\} \cap \pi_{k}^{-1}(V) .
\end{aligned}
$$

The diagram

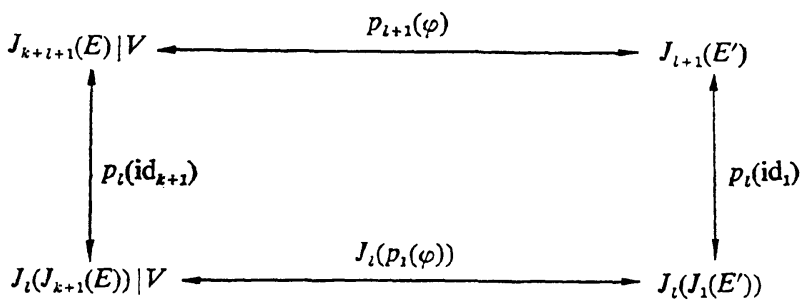

commutes by Proposition 4.3 ; since $p_{l}\left(\mathrm{id}_{1}\right) \circ j_{l+1}\left(s^{\prime}\right)=j_{l}\left(j_{1}\left(s^{\prime}\right)\right)$, we clearly have

$$
\begin{aligned}
R_{k+l+1} \cap \pi_{k}^{-1}(V) & =\operatorname{Ker}_{j_{l+1}\left(s^{\prime}\right)} p_{l+1}(\varphi) \\
& =\left\{\operatorname{Ker}_{j_{l}\left(j_{1}\left(s^{\prime}\right)\right)} J_{l}\left(p_{1}(\varphi)\right)\right\} \cap \pi_{k}^{-1}(V) .
\end{aligned}
$$

To complete the proof, we need only to show that

$$
\begin{aligned}
& \left\{\operatorname{Ker}_{j_{l}\left(j_{1}\left(s^{\prime}\right)\right)} J_{l}\left(p_{1}(\varphi)\right)\right\} \cap \pi_{k}^{-1}(V) \\
& \quad=\left\{\operatorname{Ker}_{j_{l}\left(j_{1}\left(s^{\prime}\right)\right)} J_{l}\left(p_{1}(\varphi)\right) \mid\left(J_{l}(\rho)\right)^{-1} J_{l}\left(V^{\prime}\right)\right\} \cap \pi_{k}^{-1}(V) .
\end{aligned}
$$

Clearly, the right-hand side is contained in the left-hand side. Let $q$ be an element belonging in the left-hand side; we wish to show that $J_{l}(\rho) q \in J_{l}\left(V^{\prime}\right)$. The diagram

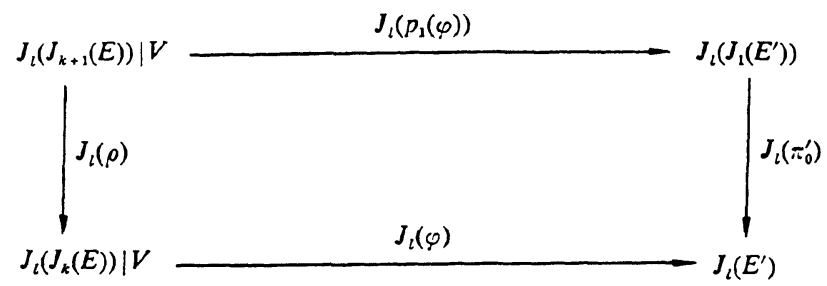

commutes by Proposition 4.2. Hence, if $x=\pi(q)$. 


$$
J_{l}(\varphi)\left(J_{l}(\rho) q\right)=J_{l}\left(\pi_{0}^{\prime}\right) j_{l}\left(j_{1}\left(s^{\prime}\right)\right)(x)=j_{l}\left(s^{\prime}\right)(x)
$$

Since $V$ is an open subset of $J_{k}(E)$, it is easily seen that $J_{l}(\rho) q \in J_{l}(V)$; the exactness of (7.2) implies that $J_{l}(\rho) q$ belongs to $J_{l}\left(V^{\prime}\right)$.

Proposition 7.2. Let $R_{k} \subset J_{k}(E)$ be a partial differential equation of order $k$ on $E$. If $g_{k+m+1}$ is a vector bundle over $R_{k}$ and $\pi_{k+m}: R_{k+m+1} \rightarrow R_{k+m}$ is surjective for $0 \leq m \leq l$, then $R_{k+m+1}$ is a submanifold of $J_{k+m+1}(E)$ and $\pi_{k+m}: R_{k+m+1} \rightarrow R_{k+m}$ is an affine sub-bundle of $\pi_{k+m}: J_{k+m+1}(E) \mid R_{k+m} \rightarrow R_{k+m}$ modeled on the vector bundle $\pi_{k}^{-1} g_{k+m+1}$ over $R_{k+m}$ induced from $g_{k+m+1}$ by $\pi_{k}: R_{k+m} \rightarrow R_{k}$, for $0 \leq m \leq l$. Moreover, the $m$-th prolongation $R_{(k+l)+m}$ of the equation $R_{k+l}$ is the same as the $(l+m)$-th prolongation of the equation $R_{k}$.

Proof. We proceed by induction on $l$. For $l=1$, the proposition holds by Proposition 7.1. Now assume that the proposition holds for $l-1$, with $l \geq 2$, and that the hypotheses of the proposition hold; then $\boldsymbol{R}_{(k+l-1)+m}$ $=R_{k+l+m-1}$. Apply Proposition 7.1 to the equation $R_{k+l-1} \subset J_{k+l-1}(E)$ and obtain $R_{k+l+m}=R_{(k+l-1)+m+1}=R_{((k+l-1)+1)+m}=R_{(k+l)+m}$ by our induction hypothesis. By Lemma 6.3 and Proposition 7.1, $R_{k+l+1}$ is an affine bundle over $R_{k+l}$ modeled on the vector bundle $\pi_{k}^{-1} g_{k+l+1}$.

Corollary 7.1. If $R_{k} \subset J_{k}(E)$ is a formally integrable partial differential equation of order $k$ on $E$, then, for each $l \geq 0, R_{k+l}$ is a submanifold of $J_{k+l}(E)$ and $\pi_{k+l}: R_{k+l+1} \rightarrow R_{k+l}$ is an affine sub-bundle of $\pi_{k+l}: J_{k+l+1}(E) \mid R_{k+l}$ $\rightarrow R_{k+l}$ modeled on the vector bundle $\pi_{k}^{-1} g_{k+l+1}$ over $R_{k+l}$ induced from $g_{k+l+1}$ by $\pi_{k}: R_{k+l} \rightarrow R_{k}$.

\section{Existence of formal solutions}

Let $R_{k} \subset J_{k}(E)$ be a partial differential equation of order $k$ on $E$. Assume that $\pi_{k}: R_{k+1} \rightarrow R_{k}$ is surjective and that $g_{k+1}$ is a vector bundle. Then the conclusion of Proposition 7.1 holds. The diagram

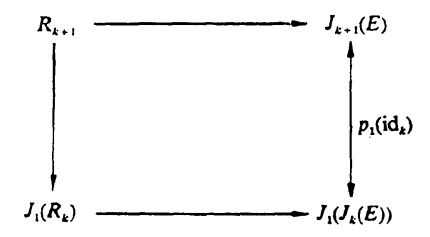

commutes; the maps of $R_{k+1}$ into $J_{1}\left(J_{k}(E)\right) \mid R_{k}$ and of $J_{1}\left(R_{k}\right)$ into $J_{1}\left(J_{k}(E)\right) \mid R_{k}$ are both monomorphisms of affine bundles over $R_{k}$. Hence the inclusion of $R_{k+1}$ into $J_{1}\left(R_{k}\right)$ is also a monomorphism of affine bundles over $R_{k}$. We let $\pi^{1}: C^{1} \rightarrow R_{k}$ be the quotient affine bundle of $J_{1}\left(R_{k}\right)$ by $R_{k+1}$, given by Proposition 3.4, and let $\rho: J_{1}\left(R_{k}\right) \rightarrow C^{1}$ be the natural epimorphism of affine bundles 
over $R_{k}$. The affine bundle $C^{1}$ is canonically isomorphic to the vector bundle $\left\{T^{*} \otimes_{R_{k}} F\left(R_{k}\right)\right\} / \delta\left(g_{k+1}\right)$ over $R_{k}$, where $\delta$ is the inclusion of $g_{k+1}$ into $T^{*} \otimes_{R_{k}} F\left(R_{k}\right)$; we shall identify these two bundles. Then the sequence

$$
O_{R_{k}} \longrightarrow R_{k+1} \stackrel{i}{\longrightarrow} J_{1}\left(R_{k}\right) \stackrel{\rho}{\longrightarrow} C^{1} \longrightarrow O_{R_{k}}
$$

is an exact sequence of affine bundles over $R_{k}$.

We recall that if 0 is the zero section of $C^{1}$ over $R_{k}$, then $J_{l}(0): J_{l}\left(R_{k}\right)$ $\rightarrow J_{l}\left(C^{1}\right)$ is the zero section of the vector bundle $J_{l}\left(\pi^{1}\right): J_{l}\left(C^{1}\right) \rightarrow J_{l}\left(R_{k}\right)$ (see Proposition 5.3).

Proposition 8.1. The map $p_{l}(\rho): J_{l+1}\left(R_{k}\right) \rightarrow J_{l}\left(C^{1}\right)$ is a morphism of fibered manifolds over $J_{l}\left(R_{k}\right)$ and

$$
R_{k+l+1}=\operatorname{Ker}_{J_{l}(0)} p_{l}(\rho) .
$$

Therefore the l-th prolongation of the first order equation $R_{k+1} \subset J_{1}\left(R_{k}\right)$ on $R_{k}$ is $R_{k+l+1}$. Moreover, if $\mathscr{S}$ denotes the sheaf of germs of solutions of $R_{k}$, the subsheaf $j_{k}(\mathscr{S}) \subset \mathscr{R}_{k}$ is the sheaf of germs of solutions of the first order equation $R_{k+1} \subset J_{1}\left(R_{k}\right)$ on $R_{k}$.

Proof. By Proposition 4.2, the diagram

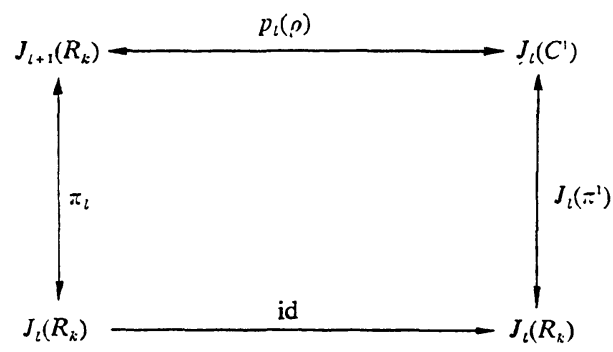

commutes, since $\rho$ is a morphism over $R_{k}$. Since $\rho$ has locally constant rank, by Proposition 4.4

$$
\operatorname{Ker}_{J_{l}(0)} J_{l}(\rho)=J_{l}\left(R_{k+1}\right)
$$

The diagram

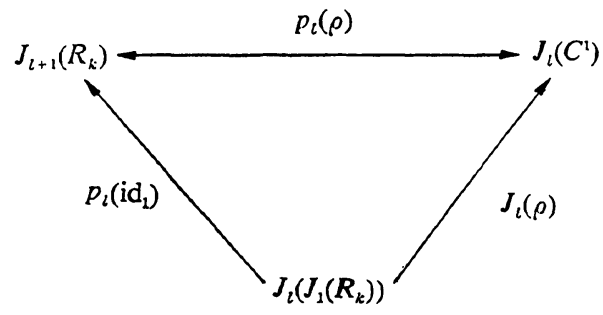


commutes by Proposition 4.3 so that

$$
\begin{aligned}
\operatorname{Ker}_{J_{l}(0)} p_{l}(\rho) & =J_{l+1}\left(R_{k}\right) \cap \operatorname{Ker}_{J_{l}(0)} J_{l}(\rho) \\
& =J_{l+1}\left(R_{k}\right) \cap J_{l}\left(R_{k+1}\right) \\
& =J_{l+1}\left(R_{k}\right) \cap J_{l}\left(R_{k+1}\right) \cap J_{k+l+1}(E) \\
& =R_{k+l+1}
\end{aligned}
$$

by Proposition 7.1, because $J_{k+l+1}(E)=J_{l+1}\left(J_{k}(E)\right) \cap J_{l}\left(J_{k+1}(E)\right)$. The remaining part of the proposition follows from the proof of Proposition 5.2 of [5].

Let $\sigma: T^{*} \otimes_{R_{k}} F\left(R_{k}\right) \rightarrow C^{1}$ denote the natural projection; then $\sigma$ is the morphism of vector bundles associated to $\rho$.

Proposition 8.2. The map $p_{l}(\rho): J_{l+1}\left(R_{k}\right) \rightarrow J_{l}\left(C^{1}\right)$ is a morphism of affine bundles over

$$
\text { id } \times p_{l-1}(\rho): J_{l}\left(R_{k}\right) \rightarrow J_{l}\left(R_{k}\right) \times_{J_{l-1}\left(R_{k}\right)} J_{l-1}\left(C^{1}\right), \quad \text { for } l \geq 1
$$

and its associated morphism of vector bundles is induced by the morphism $\sigma_{l}=\sigma \circ \Delta_{l, 1}: S^{l+1} T^{*} \otimes_{R_{k}} F\left(R_{k}\right) \rightarrow S^{l} T^{*} \otimes_{R_{k}} C^{1}$ over $R_{k}$.

Proof. By Propositions 8.1 and 5.6, $p_{l}(\rho)$ is a morphism of fibered manifolds over id $\times p_{l-1}(\rho)$ and a morphism of affine bundles over $p_{l-1}(\rho)$ whose associated morphism of vector bundles is induced by the map

$$
\rho_{*} \circ \Delta_{l, 1}: S^{l+1} T^{*} \otimes_{J_{1}\left(R_{k}\right)} F\left(J_{1}\left(R_{k}\right)\right) \rightarrow S^{l} T^{*} \otimes_{C^{1}} F\left(C^{1}\right)
$$

over $\rho$. By Proposition 5.3, it is clear that $p_{l}(\rho)$ is a morphism of affine bundles over id $\times p_{l-1}(\rho)$ whose associated morphism of vector bundles is determined by $\rho_{*} \circ \Delta_{l, 1}$. This vector bundle morphism is induced by $\sigma_{l}=\sigma \circ \Delta_{l, 1}$, because, by Proposition 3.7, the diagram

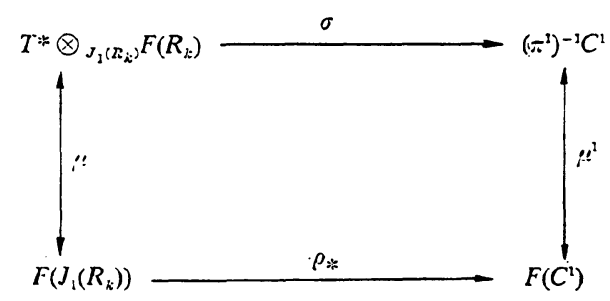

commutes.

Setting $\sigma_{0}=\sigma$, it follows from Lemma 6.3 that $g_{k+l}$ is the kernel of $\sigma_{l}$. Consider the family of vector spaces over $R_{k}$ 


$$
C^{2}=\left\{\wedge^{2} T^{*} \otimes_{R_{k}} F\left(R_{k}\right)\right\} / \delta\left(T^{*} \otimes_{R_{k}} g_{k+1}\right)
$$

whose projection onto $R_{k}$ we denote by $\pi^{2}$; let $\sigma$ be the projection of $\wedge^{2} T^{*} \otimes_{R_{k}} F\left(R_{k}\right)$ onto $C^{2}$. Then $C^{2}$ is a vector bundle if and only if $g_{k+2}$ is a vector bundle over $R_{k}$, since the sequence

$$
0 \longrightarrow g_{k+2} \stackrel{\delta}{\longrightarrow} T^{*} \otimes_{R_{k}} g_{k+1} \stackrel{\delta}{\longrightarrow} \wedge^{2} T^{*} \otimes_{R_{k}} F\left(R_{k}\right) \stackrel{\sigma}{\longrightarrow} C^{2} \longrightarrow 0
$$

is exact by Lemma 6.2. Let

$$
\tau: T^{*} \otimes_{R_{k}} C^{1} \longrightarrow C^{2}
$$

be the epimorphism of vector bundles over $R_{k}$ induced by the multiplication map from $T^{*} \otimes T^{*}$ to $\wedge^{2} T^{*}$.

Lemma 8.1. The sequence

$$
0 \longrightarrow g_{k+2} \longrightarrow S^{2} T^{*} \otimes_{R_{k}} F\left(R_{k}\right) \stackrel{\sigma_{1}}{\longrightarrow} T^{*} \otimes_{R_{k}} C^{1} \stackrel{\tau}{\longrightarrow} C^{2} \longrightarrow 0
$$

is exact.

Proof. Consider the commutative exact diagram:

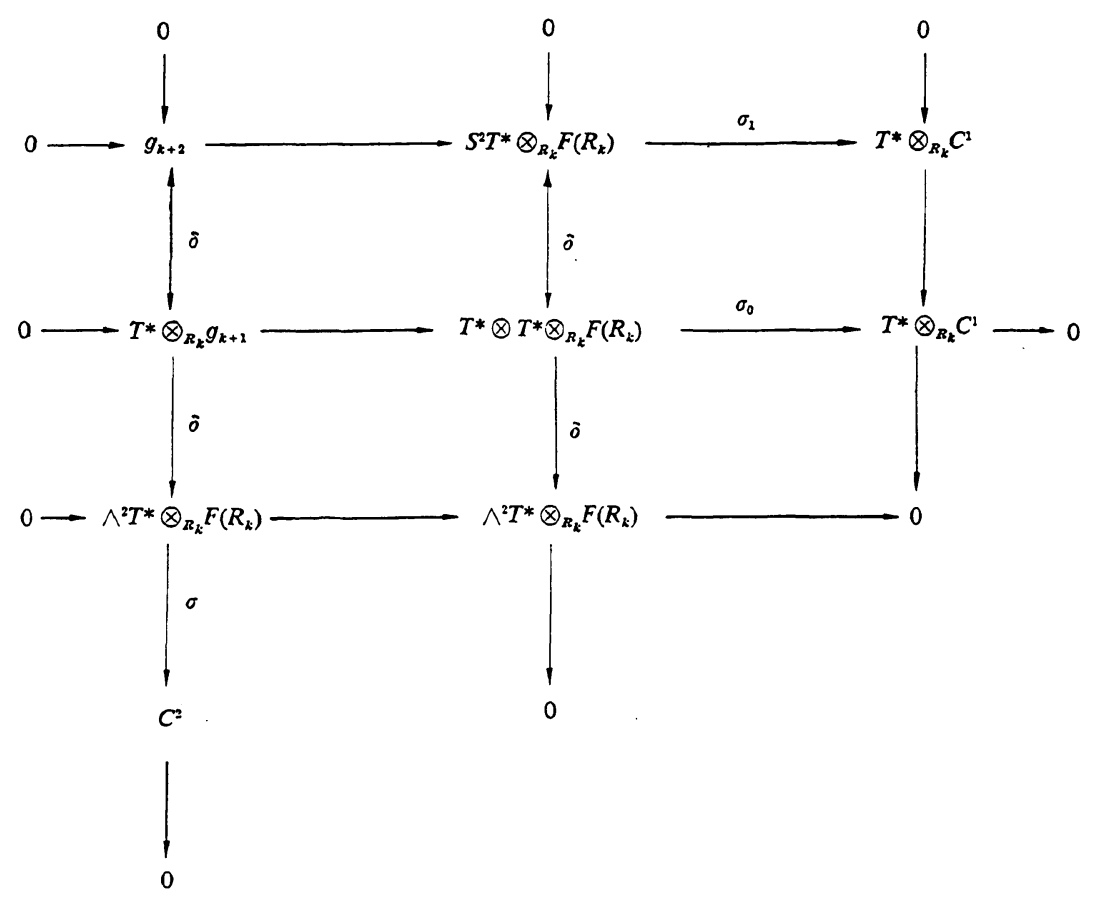


The map $\sigma \cdot \delta \cdot \sigma_{0}^{-1}: T^{*} \otimes_{R_{k}} C^{1} \rightarrow C^{2}$ is well-defined and induces an isomorphism between the cokernel of $\sigma_{1}: S^{2} T^{*} \otimes_{R_{k}} F\left(R_{k}\right) \rightarrow T^{*} \otimes_{R_{k}} C^{1}$ and $C^{2}$, which is easily seen to be, by the diagram, the map $-\tau$.

Proposition 8.3. There is a morphism over $R_{k}$

$$
\kappa=\kappa\left(R_{k}\right): R_{k+1} \longrightarrow C^{2}
$$

the curvature of $R_{k}$ such that the sequence

$$
R_{k+2} \stackrel{\pi_{k+1}}{\longrightarrow} R_{k+1} \stackrel{\kappa}{\underset{0 \circ \pi_{k}}{\longrightarrow}} C^{2}
$$

is exact.

Proof. Consider the commutative diagram

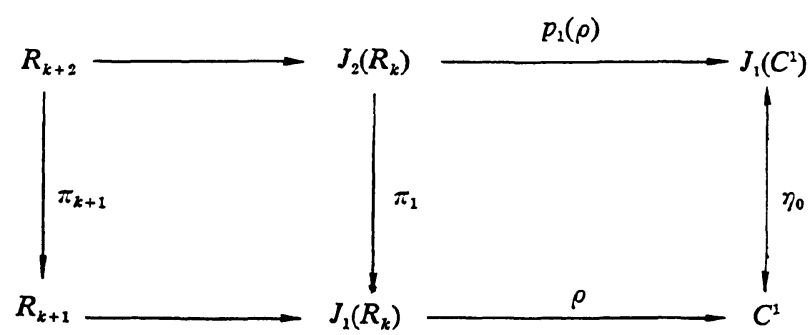

where $\eta_{0}: J_{1}\left(C^{1}\right) \rightarrow C^{1}$ is the natural projection, and the exact sequence (8.1).

Let $p$ be an element of $R_{k+1}$, and $q$ any element of $J_{2}\left(R_{k}\right)$ satisfying $\pi_{1}(q)=p$. Then

$$
\eta_{0}\left(p_{1}(\rho) q\right)=\rho(p)=0\left(\pi_{k}(p)\right) ;
$$

hence by Proposition 5.3, there exists a unique element $\varepsilon^{-1} p_{1}(\rho) q$ of $T^{*} \otimes_{R_{k}} C^{1}$ such that

$$
\varepsilon\left(p, \varepsilon^{-1} p_{1}(\rho) q\right)=p_{1}(\rho) q .
$$

We claim that the element $\kappa\left(R_{1}\right) p=\tau \varepsilon^{-1} p_{1}(\rho) q$ of $C^{2}$ depends only on $p$. Indeed, if $q_{1}$ is another element of $J_{2}\left(R_{k}\right)$ satisfying $\pi_{1}\left(q_{1}\right)=p$, then by Proposition 8.1,

$$
J_{1}\left(\pi^{1}\right) p_{1}(\rho) q_{1}=J_{1}\left(\pi^{1}\right) p_{1}(\rho) q=p .
$$

Now $q_{1}-q$ belongs to $S^{2} T^{*} \otimes_{R_{k}} F\left(R_{k}\right)$ and by Proposition 8.2 , we have

$$
\sigma_{1}\left(q_{1}-q\right)+p_{1}(\rho) q=p_{1}(\rho) q_{1}
$$

where the left-hand side is defined in terms of the map (5.6), with $W=C^{1}$, $E=R_{k}$. Therefore by Lemma 5.3, we obtain 


$$
\sigma_{1}\left(q_{1}-q\right)=\varepsilon^{-1}\left(p_{1}(\rho) q_{1}-p_{1}(\rho) q\right)
$$

Since $\varepsilon: T^{*} \otimes_{J_{1}\left(R_{k}\right)} C^{1} \rightarrow J_{1}\left(C^{1}\right)$ is a morphism of vector bundles over $J_{1}\left(R_{k}\right)$ and since $\eta_{0}\left(p_{1}(\rho) q_{1}\right)=\eta_{0}\left(p_{1}(\rho) q\right)=0\left(\pi_{k}(p)\right)$, we conclude that

$$
\sigma_{1}\left(q_{1}-q\right)=\varepsilon^{-1} p_{1}(\rho) q_{1}-\varepsilon^{-1} p_{1}(\rho) q .
$$

Because $\tau \circ \sigma_{1}=0$, we obtain

$$
\tau \varepsilon^{-1} p_{1}(\rho) q_{1}=\tau \varepsilon^{-1} p_{1}(\rho) q .
$$

Hence $\kappa\left(R_{k}\right): R_{k+1} \rightarrow C^{2}$ is a well-defined map. If $C^{2}$ is a vector bundle, then $\kappa\left(R_{k}\right)$ is in fact a morphism of fibered manifolds over $R_{k}$.

Now, if $q \in R_{k+2}$, then $\pi_{k+1}(q)=p$ belongs to $R_{k+1}$ and $p_{1}(\rho) q=J_{1}(0)(p)$ by Proposition 8.1; hence $\varepsilon^{-1} p_{1}(\rho) q=0\left(\pi_{k}(p)\right)$ by Proposition 5.3, (iv), and so $\kappa\left(R_{k}\right) p=0$. Conversely, if $p \in R_{k+1}$ satisfies $\kappa\left(R_{k}\right) p=0$, let $q$ be an element of $J_{2}\left(R_{k}\right)$ such that $\pi_{1}(q)=p$; then $\tau \varepsilon^{-1} p_{1}(\rho) q=0$. By the exactness of the sequence (8.1), there exists some element $a \in S^{2} T^{*} \otimes_{R_{k}} F\left(R_{k}\right)$ such that

$$
\varepsilon^{-1} p_{1}(\rho) q=\sigma_{1} a
$$

or

$$
p_{1}(\rho) q=\varepsilon\left(p, \sigma_{1} a\right) .
$$

Hence by Propositions 8.2 and 5.3 and Corollary 5.1,

$$
\begin{aligned}
p_{1}(\rho)((-a)+q) & =\sigma_{1}(-a)+p_{1}(\rho) q \\
& =\sigma_{1}(-a)+\left(J_{1}(0)(p)+p_{1}(\rho) q\right) \\
& =\left(\sigma_{1}(-a)+J_{1}(0)(p)\right)+p_{1}(\rho) q \\
& =\varepsilon\left(p, \sigma_{1}(-a)\right)+\varepsilon\left(p, \sigma_{1} a\right) \\
& =J_{1}(0)(p) .
\end{aligned}
$$

By Proposition 8.1, it follows that the element $(-a)+q$ of $J_{2}\left(R_{k}\right)$ satisfying $\pi_{1}((-a)+q)=p$ belongs to $R_{k+2}$.

Proposition 8.4. The image of $k$ lies in the family of subspaces over $R_{k}$

$$
H^{k, 2}=\frac{\operatorname{Ker}\left\{\delta: \wedge^{2} T^{*} \otimes_{R_{k}} g_{k} \rightarrow \wedge^{3} T^{*} \otimes S^{k-1} T^{*} \otimes_{R_{k}} F(E)\right\}}{\delta\left(T^{*} \otimes_{R_{k}} g_{k+1}\right)}
$$

of $C^{2}$.

Proof. Let $C^{j}(E)$ be the vector bundle

$$
\left\{\wedge^{j} T^{*} \otimes_{J_{k}(E)} F\left(J_{k}(E)\right)\right\} / \delta\left(\wedge^{j-1} T^{*} \otimes S^{k+1} T^{*} \otimes_{J_{k}(E)} F(E)\right)
$$

over $J_{k}(E)$, for $j=1,2$. Consider the exact commutative diagram (8.2) 


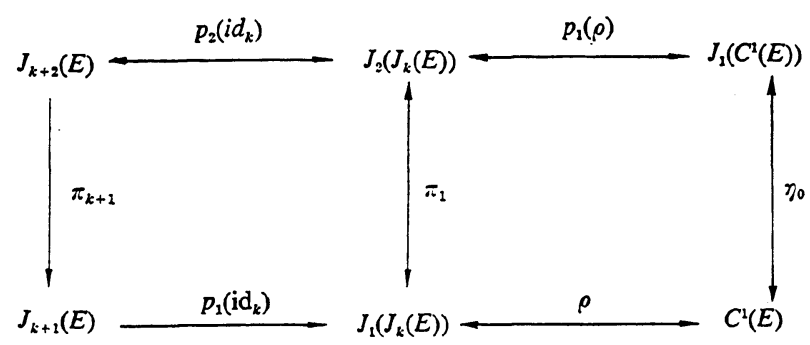

and the exact sequence (8.1)

$$
\begin{aligned}
& 0 \longrightarrow S^{k+2} T^{*} \otimes_{J_{k}(E)} F(E) \longrightarrow S^{2} T^{*} \otimes_{J_{k}(E)} F\left(J_{k}(E)\right) \\
& \stackrel{\sigma_{1}}{\longrightarrow} T^{*} \otimes_{J_{k}(E)} C^{1}(E) \stackrel{\tau}{\longrightarrow} C^{2}(E) \longrightarrow 0
\end{aligned}
$$

of vector bundles over $J_{k}(E)$ corresponding to the equation $J_{k}(E)$ of order $k$ on $E$. By Proposition 8.3, we obtain a map $\kappa\left(J_{k}(E)\right): J_{k+1}(E) \rightarrow C^{2}(E)$, which must be the zero map over $J_{k}(E)$, since $\pi_{k+1}: J_{k+2}(E) \rightarrow J_{k+1}(E)$ is an epimorphism. The exact commutative diagram

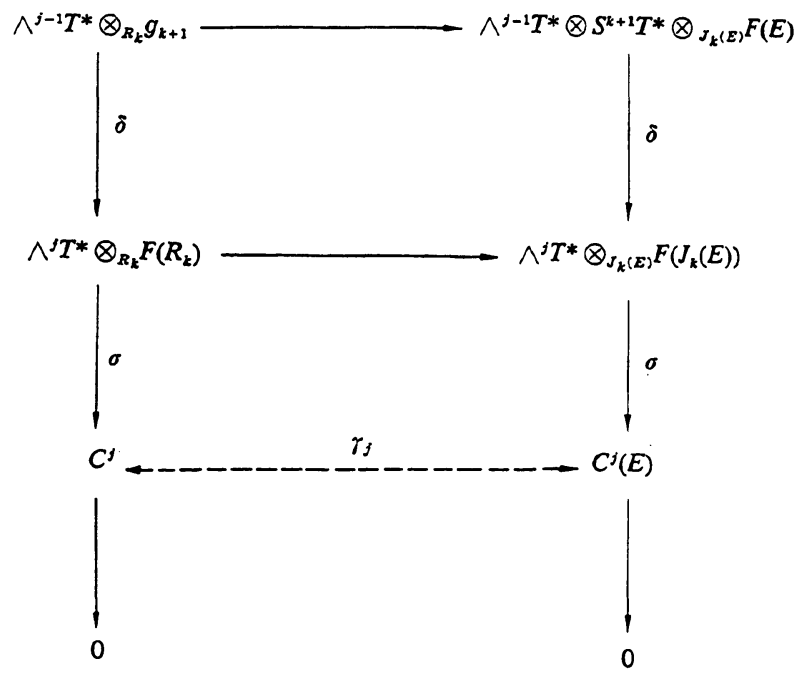

induces a morphism $\gamma_{j}: C^{j} \rightarrow C^{j}(E)$ over the inclusion of $R_{k}$ into $J_{k}(E)$ and which is therefore a morphism of fibered manifolds over $X$, for $j=1,2$. The following three-dimensional diagram clearly commutes: 


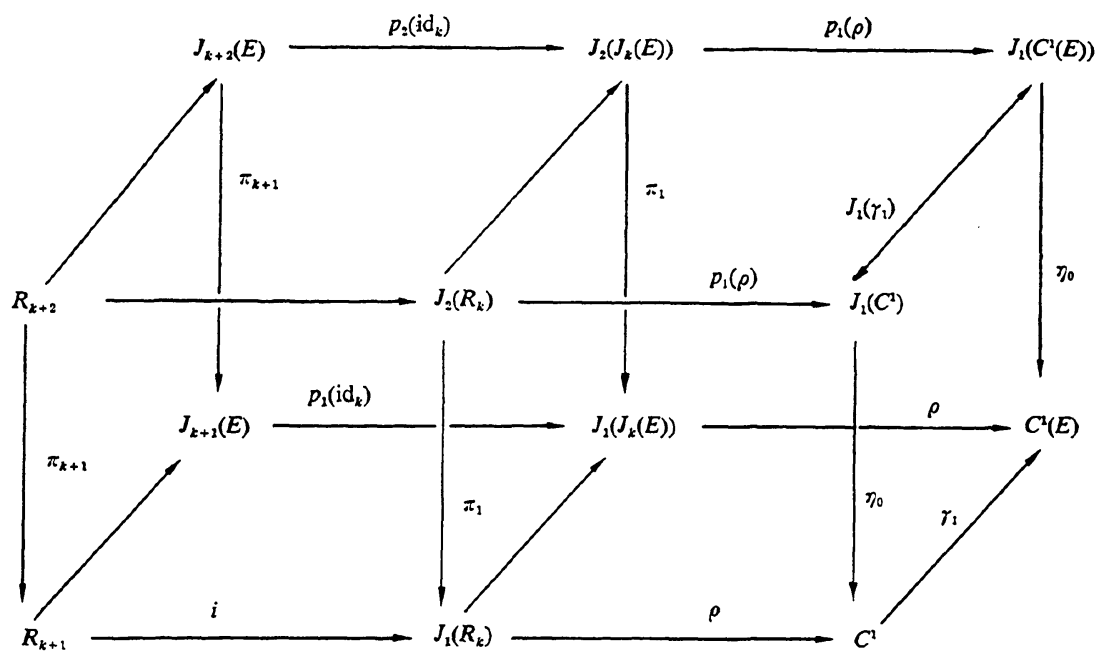

as does the diagram:

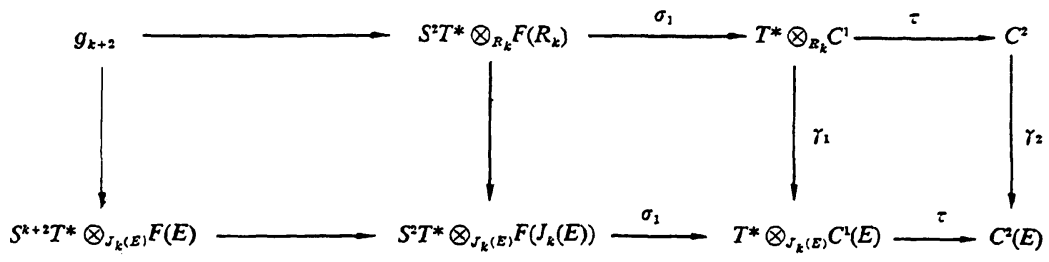

Therefore, by Propositions 8.3, 5.3, 5.4 and 3.7 the diagram

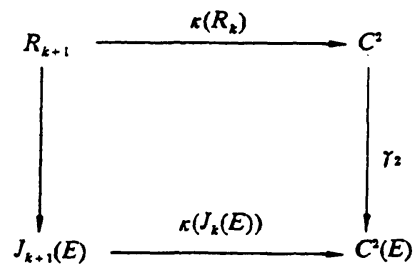

commutes, and so by the exactness and commutativity of diagram (8.3) with $j=2$, the image of $k\left(R_{k}\right)$ must lie in the family of vector spaces

$$
\left\{\wedge^{2} T^{*} \otimes_{R_{k}} F\left(R_{k}\right)\right\} \cap \delta\left(T^{*} \otimes S^{k+1} T^{*} \otimes_{R_{k}} F(E)\right) / \delta\left(T^{*} \otimes_{R_{k}} g_{k+1}\right)
$$

over $R_{k}$ which is precisely $H^{k, 2}$ by Lemma 6.1 .

Theorem 8.1. Let $R_{k} \subset J_{k}(E)$ be a partial differential equation of order $k$ 
on $E$ such that $\pi_{k}: R_{k+1} \rightarrow R_{k}$ is surjective. If $g_{k}$ is 2-acyclic, and $g_{k+1}$ is a vector bundle over $R_{k}$, then $R_{k}$ is formally integrable.

Proof. Lemma 6.5 implies that $g_{k+l}$ is a vector bundle for $l \geq 1$. We now proceed by induction on $l$. Assume that $l \geq 0$ and that $\pi_{k+m}: R_{k+m+1} \rightarrow R_{k+m}$ is a surjective map for $0 \leq m \leq l$. By Proposition 7.2, we can apply Propositions 8.3, 8.4 to the equation $R_{k+l} \subset J_{k+l}(E)$ and we obtain, by Proposition 7.2 and Lemma 6.3, an exact sequence

$$
R_{k+l+2} \stackrel{\pi_{k+l+1}}{\longrightarrow} R_{k+l+1} \stackrel{\kappa\left(R_{k+l}\right)}{\longrightarrow} \pi_{k}^{-1} H^{k+l, 2}
$$

where $\pi_{k}^{-1} H^{k+l, 2}$ is the family of vector spaces over $R_{k+l}$ induced from $H^{k+l, 2}$ by $\pi_{k}: R_{k+l} \rightarrow R_{k}$. Since $g_{k}$ is 2-acyclic, it follows that $H^{k+l, 2}=0$ and hence that the map $\pi_{k+l+1}: R_{k+l+2} \rightarrow R_{k+l+1}$ is surjective.

If the dimensions of all components of $E$ are the same, then there exists an integer $k_{0}>k$ depending on $n, k$ and the dimension of $E$ such that $g_{k_{0}}$ is involutive by Lemma 6.4, so by Proposition 7.2 we have clearly also proved

Theorem 8.2. If the dimensions of all components of $E$ are the same, and $R_{k} \subset J_{k}(E)$ is a partial differential equation of order $k$ on $E$, then there exists an integer $k_{0}>k$ depending only on $n, k$ and the dimension of $E$ such that, if $g_{k+l+1}$ is a vector bundle over $R_{k}$ and $\pi_{k+l}: R_{k+l+1} \rightarrow R_{k+l}$ is surjective, for $0 \leq l \leq k_{0}-k$, then $R_{k}$ is formally integrable.

\section{Existence of analytic solutions}

Assume that $X$ is a real analytic manifold and that the fibered manifold $\pi: E \rightarrow X$ is real analytic; we say that a partial differential equation $R_{k}$ of order $k$ on $E$ is analytic if it is an analytic fibered submanifold of $J_{k}(E)$.

Theorem 9.1. Let $R_{k}$ be an analytic partial differential equation of order $k$ on $E$ which is formally integrable. Then, given $p \in R_{k+l}$, with $\pi(p)=x \in X$, there exists an analytic solution $s$ of the equation $R_{k}$ over a neighborhood of $x$ such that $j_{k+l}(s)(x)=p$.

Proof. Consider the diagram, for $l \geq 1$,

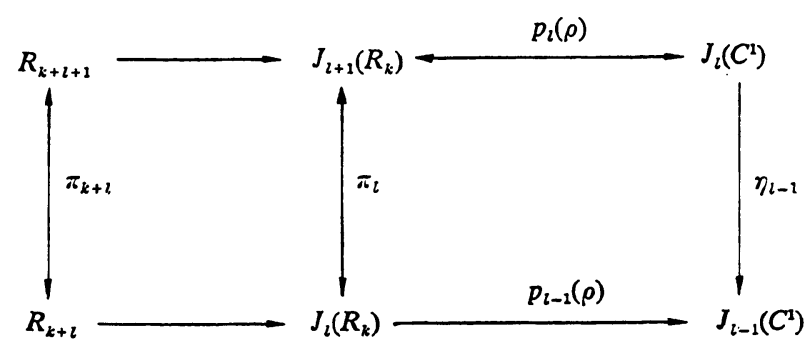

where $\eta_{l-1}$ is the natural projection, and the exact sequence 


$$
0 \longrightarrow g_{k+l+1} \longrightarrow S^{l+1} T^{*} \otimes_{R_{k}} F\left(R_{k}\right) \stackrel{\sigma_{l}}{\longrightarrow} S^{l} T^{*} \otimes_{R_{k}} C^{1}
$$

of vector bundles over $R_{k}$. Since $\pi_{k+l}: R_{k+l+1} \rightarrow R_{k+l}$ is surjective, by a diagram chase involving (9.1) and (9.2) similar to the one given in Proposition 8.3 , it is easily seen that $\sigma_{l}\left(S^{l+1} T^{*} \otimes_{R_{k}} F\left(R_{k}\right)\right)$ is equal to the set of all elements of the form $\varepsilon^{-1} p_{l}(\rho) q$, with $q \in J_{l+1}\left(R_{k}\right)$ satisfying $\pi_{l}(q) \in R_{k+l}$. Hence, restricting our attention to the fibers of all fibered manifolds at $x \in X$, using Spencer's estimate (see L. Ehrenpreis, V. W. Guillemin, and S. Sternberg [4], and W. J. Sweeney [10]) and a diagram chase involving (9.1) and (9.2) similar to the preceding one, one obtains by Proposition 8.1 and Corollary 7.1 the desired analytic solution $s$ over a neighborhood of $x$.

\section{References}

[1] R. Bott, Notes on the Spencer resolution, Mimeographed notes, Harvard University, 1963.

[2] N. Bourbaki, Algèbre linéaire, Hermann, Paris, 1962.

[3] E. Cartan, Les systèmes différentiels extérieurs et leurs applications géométriques, Hermann, Paris, 1945.

[4] X. Ehrenpreis, V.W. Guillemin \& S. Sternberg, On Spencer's estimate for ס-Poincaré, Ann. of Math. 82 (1965) 128-138.

[5] H. Goldschmidt, Existence theorems for analytic linear partial differential equations, Ann. of Math. 86 (1967) 246-270.

[6] M. Kuranishi, Lectures on exterior differential systems, Tata Institute of Fundamental Research, Bombay, 1962.

[7] D. G. Quillen, Formal properties of over-determined systems of linear partial differential equations, Ph.D. thesis, Harvard University, 1964.

[8] D. C. Spencer, Deformations of structures on manifolds defined by transitive, continuous pseudogroups, I-II, Ann. of Math. 76 (1962) 306-445.

[9] S. Sternberg, Notes on partial differential equations, Mimeographed notes, Harvard University, to appear.

[10] W. J. Sweeney, The o-Poincaré estimate, Pacific J. Math. 20 (1967) 559-570.

HARVARD UNIVERSITY STANFORD UNIVERSITY 
